\title{
Elevated outcome-anticipation and outcome-evaluation ERPs associated with a greater preference for larger-but-delayed rewards
}

\author{
Narun Pornpattananangkul ${ }^{1,2}$ - Ajay Nadig ${ }^{1} \cdot$ Storm Heidinger $^{1} \cdot$ Keegan Walden $^{1}$. \\ Robin Nusslock ${ }^{1}$
}

Published online: 21 February 2017

(C) Psychonomic Society, Inc. 2017

\begin{abstract}
Although waiting for a reward reduces or discounts its value, some people have a stronger tendency to wait for larger rewards and forgo smaller-but-immediate rewards. This ability to delay gratification is captured by individual differences in so-called intertemporal choices in which individuals are asked to choose between larger-but-delayed versus smaller-but-immediate rewards. The current study used event-related potentials (ERPs) to examine whether enhancement in two neurocognitive processes, outcome anticipation and outcome evaluation, modulate individual variability in intertemporal responses. After completing a behavioral intertemporal choice task, 34 participants performed an ERP gambling task. From this ERP task, we separately examined individual differences in outcome anticipation (stimulus-preceding negativity; SPN), early outcome valuation (feedback-related negativity; FRN), and late outcome evaluation (P3). We observed that both elevated outcome-anticipation (SPN) and late outcome-evaluation (P3) neural processes predicted a stronger preference toward larger-but-delayed rewards. No relationship was observed between intertemporal responses and early outcome evaluation
\end{abstract}

Ajay Nadig and Storm Heidinger contributed equally to this work.

Electronic supplementary material The online version of this article (doi:10.3758/s13415-017-0501-4) contains supplementary material, which is available to authorized users.

Narun Pornpattananangkul

nonnarun@u.northwestern.edu; psypn@nus.edu.sg

1 Department of Psychology, Northwestern University, Evanston, IL, USA

2 Department of Psychology, Faculty of Arts and Social Sciences, National University of Singapore, Singapore, Singapore
(FRN), indicating that the relationship between outcome evaluation and intertemporal responses was specific to the late outcome-evaluation processing stream. Moreover, multiple regression analyses indicated that the SPN and P3 independently modulate individual differences in intertemporal responses, suggesting separate mechanisms underlie the relationship between these two neurocognitive processes and intertemporal responses. Accordingly, we identify two potential neurocognitive modulators of individual variability in intertemporal responses. We discuss the mechanisms underlying these modulators in terms of anticipation-related processing (SPN) and a saliency bias toward gain (compared to loss) outcomes (P3).

Keywords Intertemporal responses $\cdot$ Stimulus-preceding negativity $\cdot$ Feedback-related negativity $\cdot \mathrm{P} 3 \cdot$ Outcome anticipation $\cdot$ Outcome evaluation

Intertemporal responses occur when people make a choice between smaller-but-immediate versus larger-but-delayed rewards (Ainslie, 1975; Frederick, Loewenstein, \& O'Donoghue, 2003; Kalenscher \& Pennartz, 2008; Peters \& Büchel, 2011; Samuelson, 1937; Schultz, 2010). While some people are more willing to wait for larger rewards and forgo smaller, more immediate rewards, others are prone to not wait and opt for the smaller but more immediate reward. Some psychologists have considered individual differences in intertemporal responses as a personality trait, given the high test-retest reliability of intertemporal responses over the course of months and years (Kirby, 2009; Odum, 2011; Ohmura, Takahashi, Kitamura, \& Wehr, 2006). On the one hand, a stronger preference toward smaller-butimmediate rewards is linked to elevated impulsiveness (Plichta $\&$ Scheres, 2014). People with personality profiles and clinical symptoms related to elevated impulsiveness (e.g., those who have obesity, gambling behaviors, drug use, and bipolar 
symptoms) often show a stronger preference toward smaller-butimmediate rewards (Ahn et al., 2011; Decker, Figner, \& Steinglass, 2014; Reynolds, 2006; Weller, Cook, Avsar, \& Cox, 2008). On the other hand, a stronger preference toward larger-butdelayed rewards is associated with an ability to delay gratification (Benningfield et al., 2014). For instance, people with behaviors and clinical symptoms related to a particularly strong tendency to delay gratification (e.g., those who have high financial savings, high educational attainment, healthy body weight, and, in the extreme, anorexia symptoms) typically display a stronger preference toward larger-but-delayed rewards (Ayduk et al., 2000; Decker et al., 2014; Ersner-Hershfield, Garton, Ballard, Samanez-Larkin, \& Knutson, 2009; Schlam, Wilson, Shoda, Mischel, \& Ayduk, 2013).

Separate lines of behavioral economics and neuroimaging research suggest that two basic neurocognitive processes, outcome anticipation and outcome evaluation, are involved in modulating individual differences in intertemporal responses (Ballard \& Knutson, 2009; Weber et al., 2007). But researchers have yet to examine whether these two processes account for shared or unique individual variability in intertemporal responses. A significant challenge of previous research attempting to disentangle the influence of outcome anticipation and outcome evaluation is the fact that these two processes occur close to each other in time. Here, we overcome this challenge by taking advantage of the high temporal resolution of event-related potentials (ERPs; Luck, 2014) to separately investigate the contributions from outcomeanticipation and outcome-evaluation processes.

\section{Individual differences in outcome anticipation}

Loewenstein and colleagues have proposed the involvement of anticipation-related processes in making intertemporal responses (Berns, Laibson, \& Loewenstein, 2007; Frederick et al., 2003; Loewenstein, 2000). They noted that, "the anticipation of an outcome can lead to physiological arousal, but does this state of anticipation enter into the decision-making process? Under certain circumstances it does....Anticipation can confer utility (or disutility) in, and of, itself" (Berns et al., 2007, p. 3). Empirically, a growing body of research suggests an association between outcome anticipation and intertemporal responses (Bartels \& Urminsky, 2011; Ersner-Hershfield et al., 2011; Ersner-Hershfield, Garton, et al., 2009; Ersner-Hershfield, Wimmer, \& Knutson, 2009; Peters \& Büchel, 2010; Pronin, Olivola, \& Kennedy, 2008; Weber et al., 2007). In one study (Weber et al., 2007), the first half of participants were told that they had been selected to receive a larger-but-delayed reward (e.g., a \$75 Amazon gift certificate in 3 months), but they had an option to opt out for a smaller-but-immediate reward (e.g., a \$50 Amazon gift certificate that day). Conversely, the second half of participants were told that they had been selected to receive a smaller-but-immediate reward, but they had an option to opt out for a larger-but-delayed reward. Weber and colleagues (2007) also had an open-ended question, asking participants to list their thoughts during the decision. The first half of participants listed more thoughts related to the future than the second half of participants did and showed a stronger preference toward the larger-but-delayed reward. This suggests that increasing the anticipation of an outcome led to enhanced preference for larger-but-delayed rewards.

Nonetheless, it is unclear whether individual differences in outcome anticipation are related to intertemporal responses. Based on the aforementioned research that manipulated anticipation-related processes (Weber et al., 2007), people who have a stronger degree of outcome anticipation may be more likely to delay gratification and, in turn, may generally prefer to wait for larger rewards. To capture individual differences in outcome-anticipation processes in the current study, we assessed an ERP component called the stimulus-preceding negativity (SPN; Brunia, van Boxtel, \& Böcker, 2011). The SPN is a negative, sustained ERP component that increases in negativity during the time leading up to an expected stimulus (Böcker, Brunia, \& Berg-Lenssen, 1994; Kotani et al., 2009). A recent $\mathrm{fMRI}$-constrained ERP localization study suggests a large network of brain areas corresponding to SPN activity, including the insula, anterior cingulate cortex, middle frontal gyri, among others (Kotani et al., 2015). The SPN is an appropriate tool to study outcome anticipation because its high temporal resolution allows researchers to separate outcome anticipation from other neurocognitive processes occurring close in time, such as motor preparation and outcome evaluation (Brunia, Hackley, van Boxtel, Kotani, \& Ohgami, 2011; Damen \& Brunia, 1987; Pornpattananangkul \& Nusslock, 2015).

Thought to underlie the unresolved expectation toward upcoming stimuli, the SPN is more negative when the expected stimuli are motivationally significant (Brunia, Hackley, et al., 2011; Kotani et al., 2003; Pornpattananangkul \& Nusslock, 2015). When playing a slot machine, for instance, people anticipate seeing the same stimuli three consecutive times. The activity of the SPN while waiting to see the third stimulus is higher when the first two stimuli are the same, compared to when the first two stimuli are different from each other (Donkers, Nieuwenhuis, \& van Boxtel, 2005). This is likely because people are more motivated to find out about the third stimulus when they still have a chance to win the slot machine. Additionally, the SPN in a gambling task is more negative after participants just won a larger reward, compared to a smaller reward, in a previous trial (Masaki, Takeuchi, Gehring, Takasawa, \& Yamazaki, 2006). Although this component indexes outcome anticipation, research has yet to examine the relationship between the SPN during anticipation of uncertain rewards and individual differences in intertemporal responses. 


\section{Individual differences in outcome evaluation}

Outcome evaluation is a neurocognitive process that assesses whether the current outcome is good or bad in a timely and accurate manner (Martin, 2012). To study outcome evaluation, EEG researchers often investigate ERPs that are locked to feedback in a task that reveals the result of one's action, such as the outcome of gambling during an established laboratory-based task, called the doors task (for reviews, see Luft, 2014; Martin, 2012; Proudfit, 2015; Walsh \& Anderson, 2012). In this task, participants choose between two closed doors, where only one of them has a reward behind it (Proudfit, 2015). They then see feedback revealing whether their choice leads to a loss or gain. Two ERP components at different time windows following feedback play an important role in outcome-evaluation processing: the feedback-related negativity $(\mathrm{FRN})^{1}$ and $\mathrm{P} 3$. While the FRN is a negative, frontocentral deflection ERP component peaking at around 250 ms post feedback onset, the $\mathrm{P} 3$ is a positive, parietal component that largely distributes later at around $300-600 \mathrm{~ms}$ post feedback onset. The FRN is believed to have a source at the anterior cingulate cortex (Holroyd, Nieuwenhuis, et al., 2004), while the $P 3$ likely has multiple sources, including areas in the medial temporal lobe (Martin, 2012). The FRN amplitude is typically more negative following bad-outcome feedback compared to good-outcome feedback, and thus, the FRN has traditionally been viewed as reflecting the binary evaluation of whether an outcome is bad or good (T. E. Baker \& Holroyd, 2009; Folstein \& Van Petten, 2008; Hajcak, Moser, Holroyd, \& Simons, 2006; Miltner, Braun, \& Coles, 1997; Walsh \& Anderson, 2012). The P3, on the other hand, has generally been linked to attentional resource allocation (Donchin \& Coles, 1988; Nieuwenhuis, Aston-Jones, \& Cohen, 2005). More specifically, it has been argued that, in the context of outcome evaluation, feedback-elicited P3 relates to attentional resources directed toward the motivational saliency of the feedback (Hu, Pornpattananangkul, \& Nusslock, 2015; Martin, 2012; Yeung \& Sanfey, 2004).

Given their relevance to binary evaluation (the FRN) and motivational saliency (the P3) of the outcome, a large body of recent ERP research has extended outcome-evaluation ERPs as indices of individual differences in reward sensitivity (for a review, see Proudfit, 2015). This line of research capitalizes on the idea that reward sensitivity is related to differential ERP reactivity to different outcomes (e.g., feedback to loss vs. gain in the gambling doors task; e.g., Bress, Foti, Kotov, Klein, \& Hajcak, 2013; Bress, Smith, Foti, Klein, \& Hajcak, 2012; Foti \& Hajcak, 2009; Foti, Kotov, Klein, \& Hajcak, 2011). In particular, a smaller difference between outcome-evaluation ERPs to

\footnotetext{
${ }^{1}$ The feedback-related negativity (FRN) is also known as feedback negativity (FN), feedback error-related negativity (fERN), medial frontal negativity (MFN), reward positivity and reward-related positivity (RewP; Proudfit, 2015).
}

loss versus gain feedback has been suggested to reflect blunted reward sensitivity, whereas a larger difference reflects enhanced reward sensitivity. Consistent with this idea, research has reported greater ERP differences to loss versus gain feedback in both FRN and P3 windows among individuals with elevated reward sensitivity (Balconi \& Crivelli, 2010; Bress et al., 2013; Foti \& Hajcak, 2012; Krämer, Büttner, Roth, \& Münte, 2008; Smillie, Cooper, \& Pickering, 2011; Van den Berg, Franken, \& Muris, 2011). These individuals include (1) people who self-report elevated reward sensitivity (e.g., sensitivity to reward, reward responsiveness, aggression and extraversion questionnaires), (2) people who score high on reward-based learning tasks, and (3) carriers of the Met allele of the COMT gene that is associated with higher tonic dopamine levels and stronger activity in reward-related neural areas during fMRI reward tasks (Chen et al., 2004; Yacubian et al., 2007). ${ }^{2}$

A link between reward sensitivity and intertemporal choices has been observed across recent studies (Ho, Mobini, Chiang, \& Bradshaw, 1999; Loewenstein, 1996; Pine et al., 2009). For instance, several fMRI studies have found a relationship between elevated reward-related neural activation in areas such as the ventral striatum (VS) and lateral orbitofrontal cortex (L-OFC), and a stronger preference toward larger-but-delayed rewards (Ballard \& Knutson, 2009; Benningfield et al., 2014; Boettiger et al., 2007; Samanez-Larkin et al., 2011). Moreover, Met-allele carriers of the COMT gene express a stronger preference toward larger-but-delayed rewards (Boettiger et al., 2007; Gianotti, Figner, Ebstein, \& Knoch, 2012; Smith \& Boettiger, 2012). Collectively, these studies suggest that individuals with elevated reward sensitivity may have a stronger ability to delay gratification, as reflected by a greater tendency to wait for larger rewards and forgo smaller-but-immediate rewards.

Despite this association between reward sensitivity and a stronger preference for larger-but-delayed rewards, it is not clear how outcome-evaluation ERPs are related to individual differences in intertemporal responses. The temporal resolution of ERPs complements the spatial resolution of fMRI studies (Benningfield et al., 2014; Boettiger et al., 2007) and allows researchers to investigate whether the relationship between individual differences in reward sensitivity and intertemporal choices is specific to early (FRN) or later (P3) outcome-evaluation processes. Over the past few years, several intertemporal studies have been conducted with outcome-evaluation ERPs as their

\footnotetext{
2 This conceptualization of reward sensitivity typically used in ERP and psychophysiological literature (i.e., based on self-report scales, reinforcement learning tasks, and genotype) does not perfectly align with the definition of reward sensitivity discussed in economics, which typically views reward sensitivity in terms of the curvature of a utility function (Pine et al., 2009). According to this view in economics, people with high reward sensitivity are those who are less likely to diminish subjective values (utility) of objective values (reward magnitude) as the magnitude of the objective values increases, and are characterized by having less concave and more linear mapping between their subjective and objective values.
} 
main focus (Blackburn, Mason, Hoeksma, Zandstra, \& El-Deredy, 2012; Cherniawsky \& Holroyd, 2013; Mason, O'Sullivan, Blackburn, Bentall, \& El-Deredy, 2012; Qu, Huang, Wang, \& Huang, 2013). Most of this research has incorporated delay information into the feedback provided (e.g., have participants gamble and then provide them with feedback that is either delayed or immediate). Compared to immediate-reward feedback, delayed-reward feedback usually elicits a more negative FRN. Given that a more-negative FRN is typically associated with loss feedback, this suggests that people evaluate a delayed reward as worse than an immediate reward (Loewenstein, 1988; Martin, 2012). However, embedding delay information into the feedback typically prevents these studies from examining individual differences in intertemporal responses, given that participants do not have the opportunity themselves to choose between delayed versus immediate rewards.

Only one study has examined the relationship between individual differences in intertemporal responses and outcome-evaluation ERPs (Cherniawsky \& Holroyd, 2013). They found that individuals who preferred smaller-but-immediate rewards had a stronger $\Delta \mathrm{FRN}$. This contradicts the proposed link between elevated reward sensitivity and a greater preference for larger-but-delayed rewards discussed thus far. However, it is difficult to interpret the results of this study given that the authors used low-gain versus high-gain feedback in calculating the $\triangle \mathrm{FRN}$ (as opposed to loss vs. gain feedback). A smaller $\triangle \mathrm{FRN}$ among individuals who preferred larger-but-delayed rewards, for instance, might indicate (1) their insensitivity to the differentiation between low-gain and high-gain outcome (hence reflecting less reward sensitivity) or (2) their stronger sensitivity to low-gain feedback (making the FRN to low-gain and high-gain feedback similar to each other, hence reflecting high reward sensitivity). Using gain and loss feedback (as opposed to high-gain and low-gain feedback) would solve this issue, given that most people would interpret loss feedback as punishment. Accordingly, this approach would enable us to interpret the FRN as reflecting the binary evaluation of good versus bad outcome (Martin, 2012). Moreover, Cherniawsky and Holroyd's (2013) study focused only on the FRN (not the P3). It is undetermined whether there is a relationship between individual differences in intertemporal responses and outcome-evaluation ERPs at the P3 window.

\section{Current study}

This study examined whether outcome-anticipation or outcome-evaluation processes modulate individual differences in intertemporal choices. Participants first completed a behavioral intertemporal choice task. They next participated in a separate ERP, gambling doors task that provided either loss or gain feedback for each trial. The doors task has been shown to (1) elicit outcome-anticipation processes, reflected by the SPN (Foti \&
Hajcak, 2012), and (2) differentiate outcome-evaluation processes at different time windows and topography: early, frontocentral (FRN) and later, posterior (P3; Foti \& Hajcak, 2012; Van den Berg et al., 2011). Based on research that manipulated anticipation processes (Weber et al., 2007), we predicted that participants who had elevated outcome-anticipation processing (as indexed by enhanced SPN activity prior to the feedback onset in the doors task) should have a stronger preference toward larger-but-delayed rewards. Similarly, based on research on reward sensitivity and intertemporal responses (Ballard \& Knutson, 2009; Benningfield et al., 2014), we predicted that participants with elevated outcome-evaluation processing, including binary evaluation motivational saliency (as indexed by enhanced FRN and P3 following feedback onset in the doors task) should also have a stronger preference toward larger-but-delayed rewards. Moreover, examining both the FRN and P3 in the same task allowed us to test which outcome-evaluation time windows were more strongly related to intertemporal responses. Lastly, examining both outcome-anticipation and outcome-evaluation ERPs allowed us to investigate unique and combined effects of outcome-anticipation and outcome-evaluation neural processes in modulating individual differences in intertemporal responses.

\section{Material and method}

\section{Participants}

Thirty-four right-handed $(<18$, Chapman Handedness Scale; Chapman \& Chapman, 1987) community participants (13 females, 19 males $^{3}$; age $M=22.97$ years, $S D=5.87$ ) were recruited from the Chicago area. They were given $\$ 20$ for participating and an additional $\$ 5$ for completing the doors task (see below). Data from six additional participants were discarded due to EEG artifact or equipment problems. Participants had no history of head injury and were not taking psychotropic medications. All participants provided informed consent prior to the experiment, and the Northwestern University institutional review board approved the present study.

\section{Behavioral measure of intertemporal responses}

Participants completed a computer-adaptive version of the intertemporal choice task using E-Prime 2.0 (Psychology Software Tools, Sharpsburg, PA; see Fig. 1a for an example trial of this task). This behavioral task was adapted from a previous paradigm that has been used to assess individual differences in intertemporal responses (Ahn et al., 2011; Rachlin, Raineri, \& Cross, 1991). We used hypothetical rewards in the intertemporal task for two reasons. First, previous studies report a similar

\footnotetext{
${ }^{3}$ Two participants did not report their gender.
} 
pattern of responses between hypothetical and real monetary rewards (Johnson \& Bickel, 2002; Lagorio \& Madden, 2005), and, second, it allowed us to assess a wide range of delays and reward amounts. At each trial, participants were told to choose between smaller-but-immediate (e.g., "\$400 now") versus larger-but-delayed (e.g., "\$800 in 1 year") choices. There were six blocks of trials. Every trial in each block had the same specific delay: 2 weeks, 1 month, 6 months, 1 year, 3 years, or 10 years. The order of blocks was fully randomized across participants. During the first trial of each block, participants made a choice between " $\$ 400$ now" versus " $\$ 800$ at [given delay]." We then adapted the subsequent choices such that the mean of the upper and lower bounds according to the choice made was used as a smaller-but-immediate choice in the following trial (Ahn et al., 2011; Du, Green, \& Myerson, 2002; Green \& Myerson, 2004). For example, if " $\$ 400$ now" was chosen over " $\$ 800$ in 1 year," it can be inferred that the participant's subjective value of the " $\$ 800$ in 1 year" was below " $\$ 400$ now." The subsequent trial would then be used to test how much below it was by having the participant choose between "\$800 in 1 year" and \$200 now," which is the mean of upper (\$400) and lower (\$0) bounds. Thus, as the task continues, the mean of the upper and lower bounds approaches the participant's subjective value of " $\$ 800$ in [given delay]." The larger-but-delayed choice was always fixed at $\$ 800$ regardless of one's prior choice. Following procedures conventionally used in studies focusing on individual-differences in intertemporal responses (Ahn et al., 2011; Du et al., 2002; Rass, Ahn, \& O'Donnell, 2015), we continued the adaptation of the smaller-but-immediate choice to the sixth trial of each block. Accordingly, the mean of the upper and lower bounds of the sixth trial was defined as the participants' subjective value of $\$ 800$ at a given delay. By completing every block, participants' subjective values of $\$ 800$ at every delay were computed.

To quantify individual differences in intertemporal responses, we computed the area under the curve (AUC) of the subjective values (Myerson, Green, \& Warusawitharana, 2001) using a customized MATLAB script (The MathWorks, Inc., Natick, MA). Specifically, we plotted each participant' subjective values of $\$ 800$ against the length of the delay. We then summed all trapezoid areas falling under the curve of this plot (see the gray area in Fig. 1b). The final value for the AUC was the proportion of this area to the total possible area. Accordingly, the range of the AUC was from 0 (steepest discounting or strongest preference for smaller-but-immediate rewards) to 1 (no discounting or strongest preference for larger-but-delayed rewards). As previously argued (Myerson et al., 2001), the AUC is an appropriate index for accessing individual differences in intertemporal responses. Given its model-free nature, the AUC does not require assumptions that every participant's discounting curve has the same shape (e.g., hyperbola, exponential or quasihyperbolic). Moreover, the AUC has a strong test-retest reliability (Myerson et al., 2001; Ohmura et al., 2006). Accordingly, this measure has been commonly used in studies that focus on a

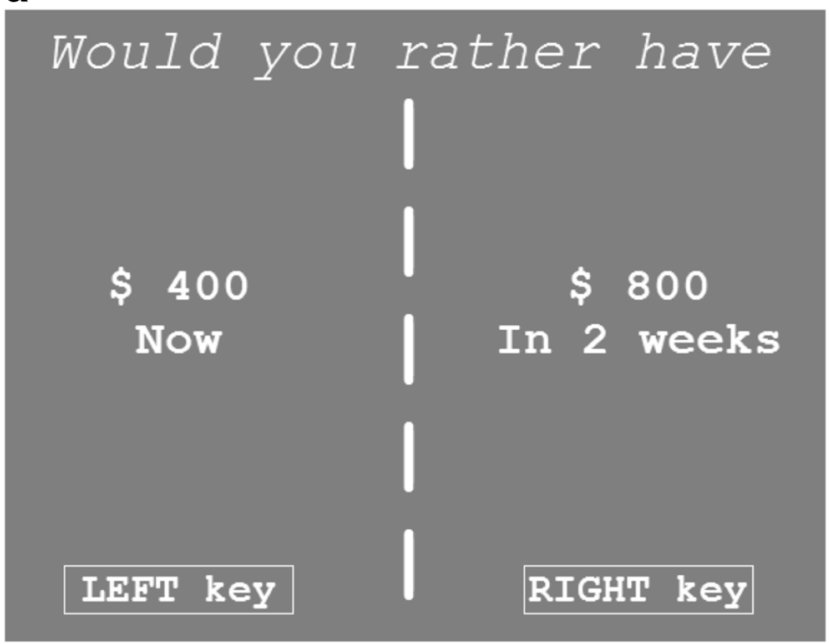

b
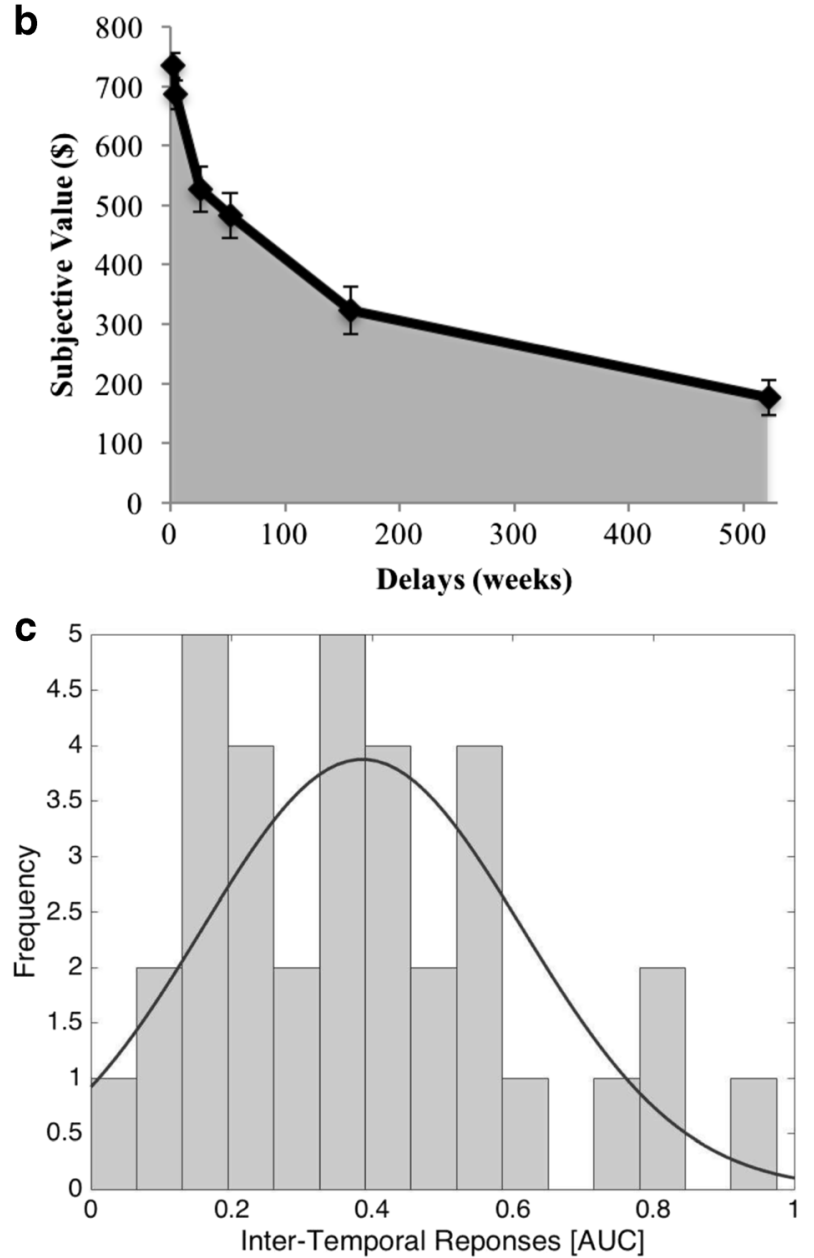

Fig. 1 The intertemporal choice task. a An example of one trial in the intertemporal choice task. b Mean subjective values of a larger-butdelayed choice (\$800) as a function of delays (in weeks). Error bars represent \pm standard error. Gray area shows the area under the curve (AUC). c Histogram of intertemporal responses (AUC). AUC is split into 15 bins. Black line indicates a normal curve

individual differences in intertemporal responses (e.g., Sellitto, Ciaramelli, \& di Pellegrino, 2010; Shamosh et al., 2008). 
ERP measure of outcome anticipation and evaluation: The doors task

Following the behavioral intertemporal choice task, participants completed the ERP doors task using the E-Prime 2.0 software (Psychology Software Tools, Sharpsburg, PA) while EEG was recorded. This ERP task was adapted from a well-established gambling/guessing task (Dunning \& Hajcak, 2007), commonly used for assessing individual differences in outcome-anticipation and outcome-evaluation processes (e.g., Van den Berg et al., 2011; Bress \& Hajcak, 2013; Foti \& Hajcak, 2009, 2012). At the beginning of each trial (see Fig. 2 for a schematic representation of the task), two pictures of a door appeared. Participants were instructed to guess which of the two doors had a prize behind it by pressing a key associated with that particular door with their right index finger. Participants earned $20 \varnothing$ for every correct guess, but lost $10 \phi$ for every incorrect guess. As previously argued (Dunning \& Hajcak, 2007), the higher magnitude of gains over losses was implemented to balance the subjective values between gains and losses (Tom, Fox, Trepel, \& Poldrack, 2007; Tversky \& Kahneman, 1992), as well as to ensure that overall earnings resulted in a positive value. After pressing the key, the pictures of the doors disappeared, and a fixation cross appeared in the middle of the screen for $2.5 \mathrm{~s}$. This relatively long interstimulus interval (ISI) was used to separate movement-related EEG activity from anticipation-related EEG activity (i.e., the SPN; Damen \& Brunia, 1994; Kotani et al., 2003). We modeled this ISI as the outcome-anticipation period. Following this ISI, participants received outcome feedback regarding their guess for a 1-s duration: (1) a green upward arrow indicated a correct guess (win 20ф); (2) a red downward arrow indicated an incorrect guess (lose 10ф). This 1-s period during which feedback was presented was modeled as the

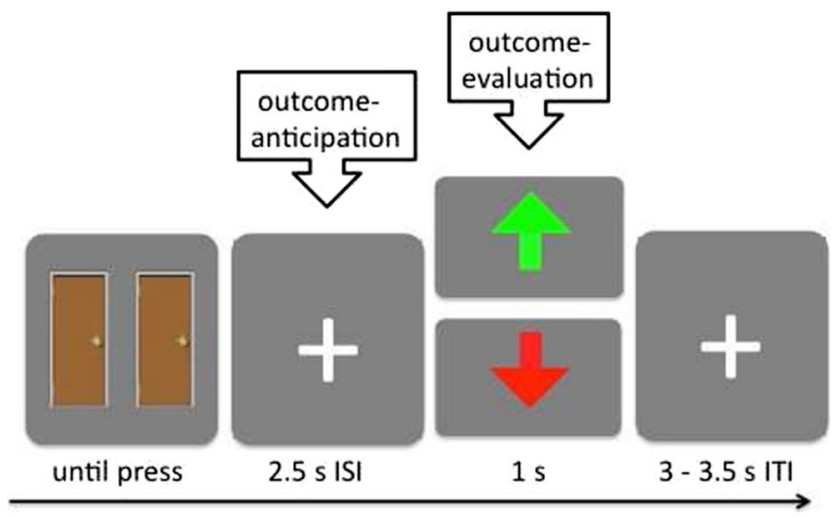

Fig. 2 The doors task. In each trial, participants first saw two doors. After they selected one of the doors by pressing a key, the doors disappeared, and there was a 2.5 -s interstimulus interval (ISI). This ISI was an outcomeanticipation period. Following the ISI is an outcome-evaluation period when participants were notified whether their guessing had led to a gain outcome (green, up arrow) or a loss outcome (red, down arrow) for $1 \mathrm{~s}$. Participants then saw another fixation cross during an intertrial interval (ITI), which was jittered between 3-3.5 s (Color figure online) outcome-evaluation period. Following feedback, there was a randomized intertrial interval (ITI) between 3 and $3.5 \mathrm{~s}$, during which a fixation cross was presented. There were 100 trials in total, consisting of five blocks of 20 trials that were separated by breaks of participant-determined length. During these breaks, participants were informed of their cumulative earnings. Unbeknownst to participants, the overall performance and earnings on the task was predetermined. Specifically, while the order of guessing outcome was fully randomized across participants, total earnings were fixed at $\$ 5$ for every participant. There were 10 practice trials before the task.

\section{Electrophysiological recording}

Continuous EEG data with a sampling rate at $500 \mathrm{~Hz}$ (DC to $100 \mathrm{~Hz}$ online; Neuroscan Inc., Charlotte, NC) were collected from inside an electromagnetic shielded booth. Twenty-three Ag/ $\mathrm{AgCl}$ scalp electrodes, including FP1/2, F7/3/z/4/8, FC3/z/4, C3/ z/4, T3/4, CP3/z/4, P3/z/4, T5/6 were used. HEOG and VEOG were recorded with four separate eye electrodes. Recordings were referenced online to a left mastoid and rereferenced off-line to linked mastoids. Impedance was kept below $5 \mathrm{k} \Omega$ and $10 \mathrm{k} \Omega$ for scalp and eye electrodes, respectively. During the off-line analyses, eye movement artifacts were first corrected with a PCA algorithm. Movement-related artifacts were removed manually. Before epoching, continuous EEG was bandpass filtered at .01-30 Hz. Epochs containing artifacts $( \pm 75 \mu \mathrm{V})$ were rejected. Data analyses was done in both NeuroScan EDIT 4.5 (Neuroscan Inc.) and EEGlab (Delorme \& Makeig, 2004).

\section{Event-related potential quantifications}

\section{Outcome-anticipation ERP: The stimulus-preceding} negativity (SPN)

The stimulus-preceding negativity (SPN) The SPN was used as an index for neural activity during outcome anticipation, which should be enhanced during the ISI, especially prior to feedback onset (Foti \& Hajcak, 2012; Kotani et al., 2003). To analyze the SPN, we first epoched 5-s response-locked EEG from -2,000 to $3,000 \mathrm{~ms}$ relative to the button press. Given that the feedback was shown 2,500 ms following the button press, the ISI in these EEG epochs was from zero to $2,500 \mathrm{~ms}$. Slow-wave artifacts were removed from these EEG epochs using a linear-detrend algorithm on a wider $(-3,000$ to $3,500 \mathrm{~ms}$ ) window (for a similar method, see K. S. Baker, Piriyapunyaporn, \& Cunnington, 2012; Goldstein et al., 2006; Pornpattananangkul \& Nusslock, 2015). Following an established guideline for SPN analyses (Brunia, van Boxtel, et al., 2011), a low-pass filter of $10 \mathrm{~Hz}$ was further applied on these epochs, resulting in .01-10 Hz data. We then performed a baseline correction using a time window prior to the button press at $-2,000$ to $-1,700 \mathrm{~ms}$. This early baseline time window was selected to avoid slow potentials created 
by preparation for pressing the button (Brunia, van Boxtel, et al., 2011). Given that ITI duration was 3,000 to $3,500 \mathrm{~ms}$ long, this baseline time window should fall during the ITI.

The SPN is characterized by a slow, negative ERP that increases in size up to the feedback onset (Brunia, van Boxtel, et al., 2011). Accordingly, we scored the SPN as a linear slope of the ERP during the 800-ms window immediately prior to feedback onset (i.e., from 1,700 to $2,500 \mathrm{~ms}$ relative to the button press). The linear slope was calculated using the following equation, slope $=\sum(x-\bar{x}) \frac{(y-\bar{y})}{\sum(x-\bar{x})}^{2}$, where $x$ is ERP amplitude in $\mu \mathrm{V}$ at each time point, and $y$ is time point (ranging from 1,700 to $2,500 \mathrm{~ms}$ ). Thus, the more negative the SPN slope, the greater the outcome-anticipation-related neural activity. We scored the SPN at a frontal midline site, Fz, following previous studies (Fuentemilla et al., 2013; Morís, Luque, \& Rodríguez-Fornells, 2013). Although the SPN at frontal sites is typically less steep than at centroparietal sites (e.g., Kotani \& Aihara, 1999), elevated SPN negativity at Fz has recently been associated with enhanced anticipation-related processes during reinforcement learning (Morís et al., 2013), and also with enhanced risk-seeking behavior, motivational state, and pleasure of obtaining rewards (Fuentemilla et al., 2013).

\section{Outcome-evaluation ERPs: The feedback-related negativity (FRN) and $P 3$}

For outcome-evaluation analyses, we epoched EEG data from -100 to $1000 \mathrm{~ms}$ relative to the feedback onset, and used the prestimulus time window (-100 to $0 \mathrm{~ms}$ ) as a baseline correction window. Here, we focused on the feedback-related negativity (FRN) and $\mathrm{P} 3$ as neural-activity indices during outcome evaluation (i.e., following the feedback onset). Unlike the SPN, the FRN and P3 do not represent slow potentials (Martin, 2012). Therefore, higher high-pass and low-pass filters $(.1-30 \mathrm{~Hz})$ were applied. This outcome-evaluation EEG epoch was averaged separately for the gain and loss feedback.

The feedback-related negativity (FRN) Compared to the P3, FRN activity usually peaks earlier and distributes more frontally on scalp topography (Martin, 2012). To score the FRN, we computed the mean activity in a 50-ms window surrounding the most negative local peak that fell between $150 \mathrm{~ms}$ and $350 \mathrm{~ms}$ after feedback onset at the frontocentral midline site, FCz, separately for the gain and loss feedback. FRN analyses focused on $\mathrm{FCz}$ following previous research (T. E. Baker \& Holroyd, 2009; Bress \& Hajcak, 2013; Cherniawsky \& Holroyd, 2013; Foti \& Hajcak, 2012; Foti et al., 2011). We then subtracted the mean-around-thepeak FRN of the gain feedback from the mean-around-the-peak FRN of the loss feedback. We refer to this value as the $\Delta$ FRN. Mean-around-the-peak was chosen to control for individual differences in ERP latency (Clayson, Baldwin, \& Larson, 2013). As such, greater negativity of the $\Delta \mathrm{FRN}$ reflects a larger difference between loss and gain FRNs, while reduced negativity reflects a smaller difference between loss and gain FRNs (Bress \& Hajcak, 2013).

The P3 Similar to the FRN, we scored the P3 separately for the gain and loss feedback, given that both gain and loss feedback typically elicit a clear, dominant positive peak distribution of the P3 at posterior, midline sites (Van den Berg et al., 2011; Pornpattananangkul \& Nusslock, 2015; Yeung \& Sanfey, 2004). Also consistent with the FRN analyses, the meanaround-the-peak method was used for the P3. Specifically, for each gain and loss waveform, we computed the mean activity in a 50-ms window surrounding the most positive local peak between $300 \mathrm{~ms}$ and $600 \mathrm{~ms}$ at a parietal, midline site, Pz, following previous research (Donchin \& Coles, 1988; Yeung \& Sanfey, 2004). To assess the difference in outcome-evaluation at the $\mathrm{P} 3$ window, we computed the $\Delta \mathrm{P} 3$ by subtracting the gainfeedback P3 value from the loss-feedback P3 value. Given that the $\mathrm{P} 3$ is a positive ERP component, more negativity of the $\Delta \mathrm{P} 3$ reflects greater $\mathrm{P} 3$ amplitude to the gain (compared to loss) feedback, while more positivity of the $\Delta \mathrm{P} 3$ reflects greater $\mathrm{P} 3$ amplitude to the loss (compared to gain) feedback.

\section{Results}

\section{Behavioral responses}

Figure $1 \mathrm{~b}$ shows the pattern of intertemporal responses. On average, the AUC was well below one $(M=.39, S D=.23)$, suggesting that, overall, participants discounted the value of delayed rewards. Moreover, visual inspection of the relationship between subjective values and delays suggests that participants discounted the subjective values of delayed rewards in a nonlinear fashion. This is consistent with theoretical models of intertemporal responses (Frederick et al., 2003; Green \& Myerson, 2004). Figure 1c shows a histogram of the AUC (skewness $=.75, S E$ $=.40$; kurtosis $=1.5, S E=.79$ ), overlaid with a normal curve. The AUC did not significantly deviate from normal, Kolmogorov-Smirnov's $D(34)=.097, p=.20$; Shapiro-Wilk's $W(34)=.947, p=.10$. Nonetheless, the histogram suggests a right-skewed distribution. Thus, to demonstrate the robustness of the inferential statistics involving the AUC, we employed both parametric (e.g., Pearson's correlation coefficient, $r$ ) and nonparametric (e.g., Spearman's rank coefficient, $r_{s}$, and ranktransformed multiple regression; Conover \& Iman, 1981) statistical tests, using the Robust Correlation and LIBRA toolboxes in MATLAB (Pernet, Wilcox, \& Rousselet, 2012; Verboven \& Hubert, 2005). Accordingly, analyses involving AUC should be protected against a nonnormal distribution and outliers. As for the behavioral responses in the doors task, the averaged reaction time was $607.91 \mathrm{~ms}(S D=286.98)$. 


\section{Event-related potentials (ERPs)}

\section{Outcome-anticipation ERP: The stimulus-preceding negativity (SPN)}

Figure 3a shows the SPN waveform at Fz and a topographic map of the SPN slope. On average, 59.97 outcome-anticipation epochs $(S D=19.13)$ were analyzed per participant. As expected, the SPN slope was negative at $\mathrm{Fz}$ during the 800 -ms window immediately prior to feedback onset $(M=-.0016 \mu \mathrm{V} / \mathrm{ms}, S D=.0024)$ and significantly lower than zero, $t(33)=-3.90, p<.0001$. The negative slope of the SPN was observed throughout electrodes along the midline (see the topographic map in Fig. 3a). The enhancement of the negative slope prior to feedback onset is consistent with SPN's characteristic waveform (Brunia, van Boxtel, et al., 2011).

More specific to the focus of our study, there was a significant negative relationship between the SPN slope at Fz and AUC, $r(32)=-.39, p=.024 ; r_{s}(32)=-.41, p=.016$; see Fig. 3b. We also found similar, significant negative correlations between AUC and SPN across the frontal/frontocentral midline electrodes (including F3, Fz, FC3, FCz, FC4, and Cz; see the topographical map in Fig. 3b). In addition to using an 800-ms window immediately prior to feedback onset to score the linear slope of the SPN, we also examined the relationship between the SPN slope and intertemporal responses at different time windows to assess the robustness of these relationships. Both a $600-\mathrm{ms}, r(32)=-.40$, $p=.02 ; r_{s}(32)=-.44, p=.009$, and a 1,000-ms, $r(32)=-.35, p=$ $.04 ; r_{s}(32)=-.40, p=.019$, window prior to feedback onset were negatively related with AUC. Thus, the relationship between the SPN slope and AUC was robust across frontocentral electrodes and time windows $(600-, 800-$ and $1,000-\mathrm{ms}$ windows prior to feedback onset). This suggests that people who had stronger outcome-anticipation processing (as indexed by a more negative SPN slope) had a stronger preference for larger-but-delayed rewards (as indexed by a larger AUC) compared to smaller-but-immediate rewards.

\section{Outcome-evaluation ERPs: The feedback-related negativity $(F R N)$ and $P 3$}

On average, 39.06 outcome-evaluation epochs $(S D=6.37)$ were analyzed for each feedback type. ${ }^{4}$ The FRN waveform at FCz and

\footnotetext{
${ }^{4}$ Note that shorter length $(1,100 \mathrm{~ms})$ in the outcome-evaluation epochs compared to $5,000 \mathrm{~ms}$ in the outcome-anticipation epochs was a reason why the number of outcome-evaluation epochs $(M=39.06 \times 2=78.12)$ was higher than the number of outcome-anticipation epochs $(M=59.97)$. That is, the longer the epoch was, the more likely that each epoch would contain artifacts (identified using the $\pm 75 \mu \mathrm{V}$ criterion) and thereby be rejected. In any case, the number of epochs for both outcome-anticipation and outcome-evaluation ERPs far exceeded the recommended numbers used in previous research (Cohen \& Polich, 1997; Kotani et al., 2003; Marco-Pallares, Cucurell, Münte, Strien, \& Rodriguez-Fornells, 2011; Ohgami, Kotani, Hiraku, Aihara, \& Ishii, 2004), ensuring the stability of all components (the SPN, FRN, and P3).
}

its topographical map are shown in Fig. 4a. Overall, we found a clear separation between the FRN and P3 at this FCz site, such that the difference between the gain and loss feedback was apparent at the FRN window, but not at the $\mathrm{P} 3$ window. Consistent with existing research (Walsh \& Anderson, 2012), the mean-around-the-peak FRN of the loss feedback $(M=-.14 \mu \mathrm{V}$, $S D=3.43)$ was more negative than the mean-around-the-peak FRN of the gain feedback $(M=1.22 \mu \mathrm{V}, S D=2.67), t(33)=$ $-3.26, p=.003$. This stronger negative deflection was maximal at $\mathrm{FCz}$, but was found across frontal midline electrodes (e.g., Fz and $\mathrm{Cz}$; see Fig. 4a). Thus, overall, participants had stronger negative deflections in the FRN time window to the loss feedback compared to the gain feedback. However, unlike the SPN, intertemporal responses (AUC) were not correlated with the $\triangle \mathrm{FRN}$ (loss FRN minus gain FRN difference score) at FCz, $r(32)=.01, p=.95 ; r_{s}(32)=-.02, p=.91$, or at any other frontal midline electrodes (see Fig. $4 \mathrm{~b}$ ). ${ }^{5}$

The P3 waveform at $\mathrm{Pz}$ and its topographical map are shown in Figure $5 \mathrm{a}$. The $\mathrm{P} 3$ at $\mathrm{Pz}$ to loss feedback $(M=$ $20.60 \mu \mathrm{V}, S D=7.77$ ) was less positive than the $\mathrm{P} 3$ to gain feedback $(M=23.32 \mu \mathrm{V}, S D=8.49), t(33)=-5.31, p<.001$. This indicates greater $\mathrm{P} 3$ amplitude to gain (compared to loss) feedback. This pattern was found throughout the parietal, midline electrodes (e.g., CP3/z/4 and P3/z/4; see Fig. 5a).

Unlike the $\triangle \mathrm{FRN}$, but similar to the SPN slope, there was a negative relationship between the $\Delta \mathrm{P} 3$ (loss $\mathrm{P} 3$ minus gain $\mathrm{P} 3$ difference score) at $\mathrm{Pz}$ and intertemporal responses (AUC), $r(32)=-.34, p=.05 ; r_{s}(32)=-.43, p=.01$; see Fig. 5 b. ${ }^{6}$

\footnotetext{
$\overline{5}$ The FRN often coincides with the P3 (Holroyd, Nieuwenhuis, Yeung, \& Cohen, 2003), making it hard to find local peaks in some individuals. Thus, there might be measurement bias when applying mean-around-the-peak of the FRN separately for the gain and loss feedback (Martin, 2012). An alternative method to isolate the FRN from the P3 is to employ a difference-waveform approach (Foti et al., 2011; Hajcak, Moser, Holroyd, \& Simons, 2007). Yet some researchers are opposed to this difference-waveform method. Luft (2014, p. 360), for instance, noted that, "[difference waves] can be risky as the difference waveform may produce components that are not present in the original waveforms" (see also Van Boxtel, Ullsperger, \& Falkenstein, 2004). As a compromise, in addition to using the mean-around-the-peak method separately for the gain and loss feedback, we also employed the difference-waveform method $(\triangle F R N)$. To compute the difference-waveform $\triangle F R N$, we first subtracted the gain ERP waveform from the loss ERP waveform, resulting in a difference waveform. To score the difference-waveform $\triangle F R N$ from this difference waveform, we next computed the mean activity in a 50-ms window surrounding the most negative local peak that fell between 150-350 ms after feedback onset at FCz (Foti et al., 2011). This difference-waveform $\triangle F R N$ was on average negative $(M=-5.30 \mu \mathrm{V}, S D=2.81)$ and significantly lower than zero, $t(33)=-10.98, p<.001$. However, similar to when the $\Delta F R N$ was computed using separate gain and loss waveforms, the difference-waveform $\Delta \mathrm{FRN}$ was not correlated with intertemporal responses (AUC), $r(32)=-.22, p$ $=.21 ; r_{s}(32)=-.29, p=.09$. This suggests that regardless of how the $\Delta \mathrm{FRN}$ was scored, the relationship between the $\triangle \mathrm{FRN}$ and AUC was not significant. ${ }^{6}$ Upon inspection of the data, however, there appears to be some potential outliers in the $\triangle \mathrm{P} 3$ data: one data point defined by a value of $1.5 \mathrm{IQR}$ away from the $25^{\text {th }}$ or $75^{\text {th }}$ percentile, and two additional data points defined by a value of two SDs away from the mean. However, using Spearman's rank coefficient $\left(r_{s}\right)$ in our correlational analyses should remedy the influence from these outliers.
} 

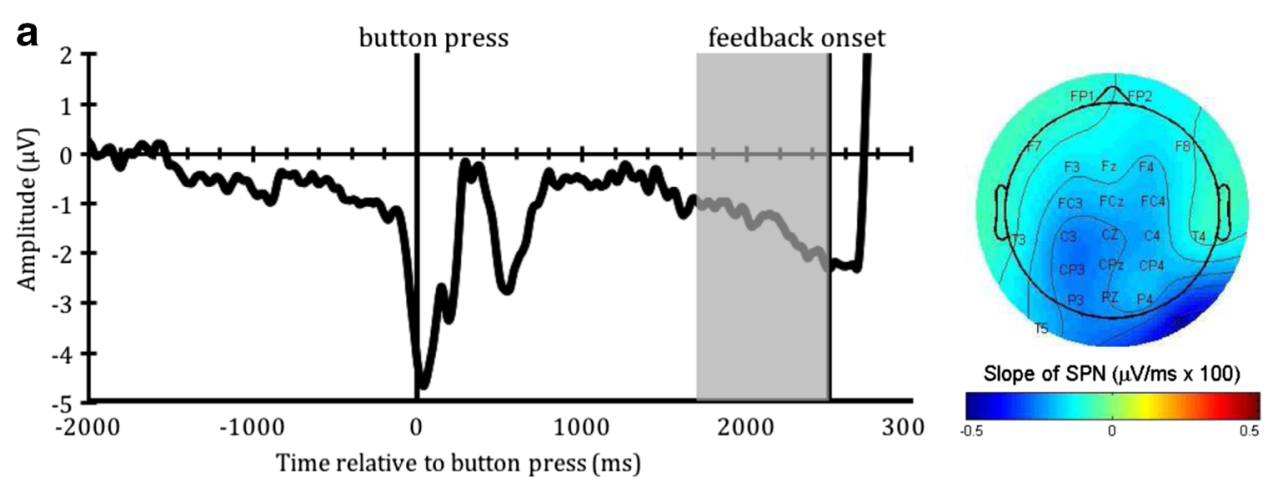

b
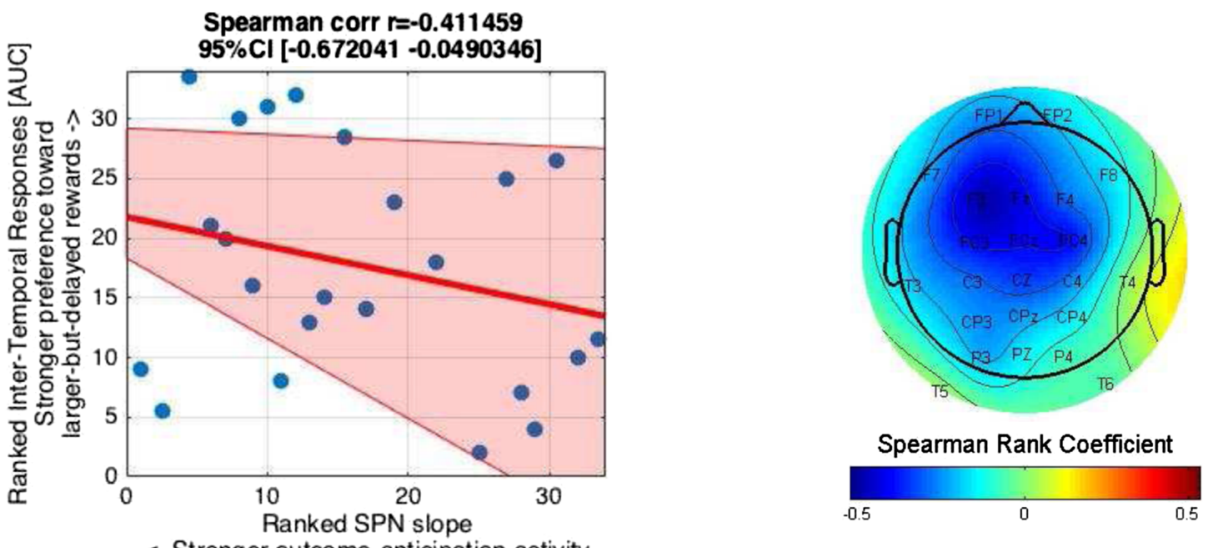

Fig. 3 The stimulus preceding negativity (SPN). a SPN waveform at Fz (left) and topographical map of the SPN slope (right). SPN slope shown in the topographical map was calculated as a linear slope of the ERP during an 800-ms window before feedback onset (i.e., from 1,700 to $2,500 \mathrm{~ms}$ after button press, indicated by a gray area in the waveform). Note, we argue that the seemingly large, positive ERP activity following feedback onset (at the edge of the window) is a deflection due to the presentation of the feedback, not a filter artifact (see Supplementary Materials p. 1-4). To demonstrate this, we expanded our epoch window to cover feedback presentation and ITI, thus showing activity elicited by the feedback (see Supplementary Figs. 1-2). We also conducted a simulation (see Supplementary Figs. 3-6) to assess the potential high-pass filter artifact (Tanner, Morgan-Short, \& Luck, 2015;

Tanner, Norton, Morgan-Short, \& Luck, 2016). Results from this simulation suggests that our high-pass filter of $.01 \mathrm{~Hz}$ had little (if any) effect on preceding activity in the window of the SPN. b Relationship between intertemporal responses (AUC) and SPN slope. A scatterplot between ranked AUC and SPN slope at Fz (left) indicates their significant relationship $(p=.016)$. The pink shaded area represent $95 \%$ bootstrapped confidence intervals around the linear regression line. The topographical map (right) depicts the relationship (Spearman rank coefficients) at different electrodes, showing significant relationships $\left(r_{s}\right.$ $<-.34, p$ 's <.05) at F3, Fz, FC3, FCz, FC4, and Cz. Note also that the more negative the SPN slope, the greater outcome-anticipation activity (Color figure online)

Moreover, this relationship was strongest at parietal sites, although this relationship was found throughout the midline electrodes (see Fig. 5b). Thus, participants who had stronger outcome-evaluation processing to the gain (compared to loss) feedback (as indexed by a more negative $\Delta \mathrm{P} 3$ ) had a stronger preference for larger-but-delayed rewards (as indexed by a larger AUC).

The Spearman's rank coefficient between the AUC and $\triangle \mathrm{P} 3$ at $\mathrm{Pz}$ was significantly different from that between the AUC and $\triangle \mathrm{FRN}$ at $\mathrm{FCz}$, as revealed by the Williams' $t$ test, $t(31)=2.20, p=.036$ (Weaver \& Wuensch, 2013; Williams, 1959). Thus, the relationship between intertemporal responses and the outcome-evaluation ERPs appears to be specific to the P3 time window.

\section{Multiple regression between intertemporal responses and outcome- anticipation/evaluation ERPS}

Since both outcome-anticipation (the SPN slope) and outcome-evaluation (the $\triangle \mathrm{P} 3$ ) ERPs were related to intertemporal responses (the AUC), we further employed multiple-regression analyses to examine unique versus shared effects of these two ERPs in predicting the AUC (see Table 1). This analysis assessed whether the SPN slope (outcome anticipation) and the $\triangle \mathrm{P} 3$ (outcome evaluation) were associated with unique versus shared variation in intertemporal responses. We first transformed the data into rank-order values before testing them in the multiple-regression model, as this rank-transformed multiple-regression method is less influenced by outliers (Conover \& Iman, 1981). We then simultaneously entered each participant's rank-transformed SPN 

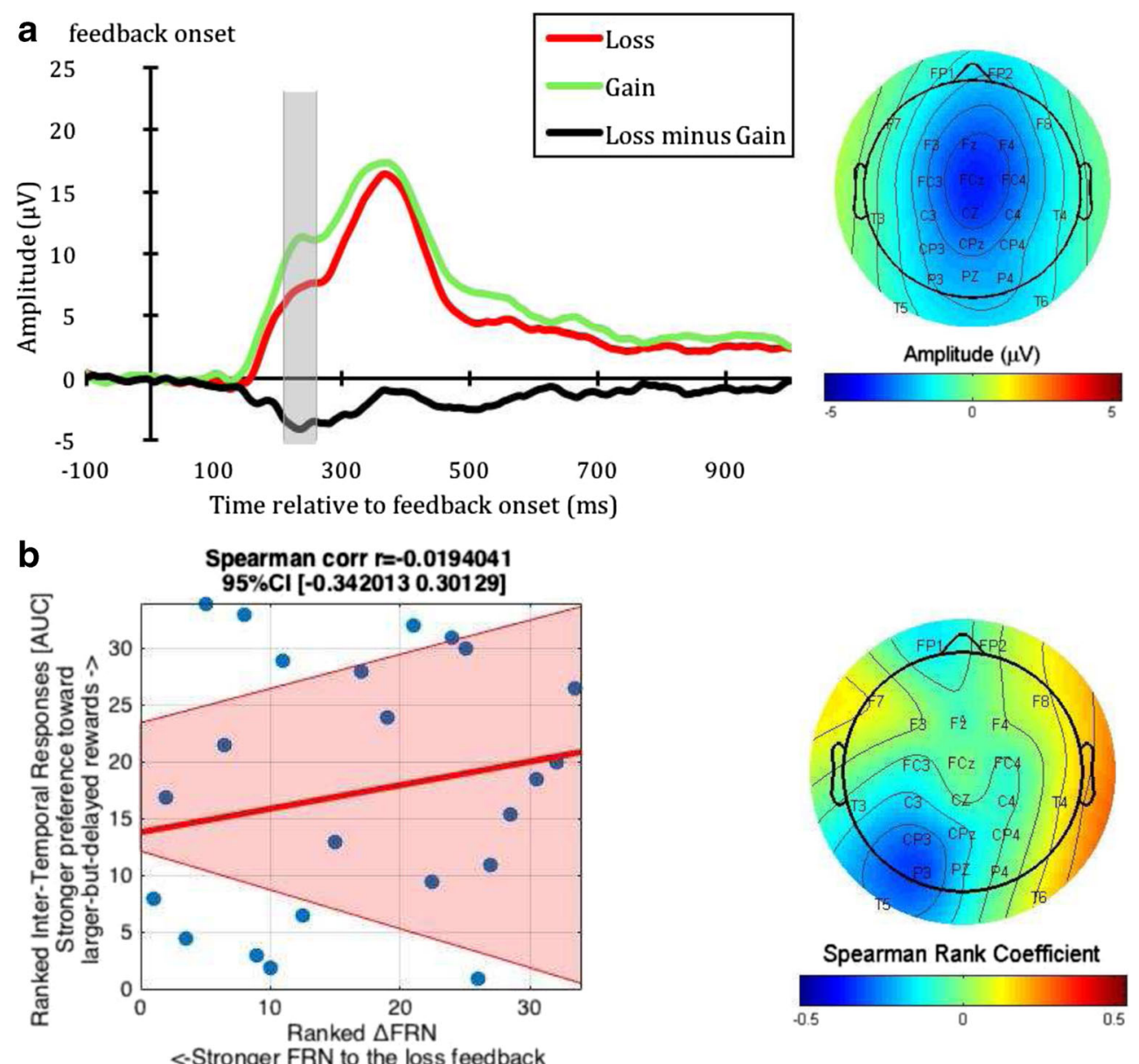

Fig. 4 The feedback-related negativity (FRN). a FRN waveform at $\mathrm{FCz}$ (left) and its topographical map (right). Topographical map shows the difference in ERP activity between the loss and gain feedback (loss minus gain) during 210 to $260 \mathrm{~ms}$ after feedback onset (indicated by the gray area in the waveform). b The (lack of) relationship between intertemporal responses (AUC) and the $\triangle \mathrm{FRN}$ (Loss FRN minus Gain $\mathrm{FRN}$ ). Scatterplot between ranked $\mathrm{AUC}$ and $\Delta \mathrm{FRN}$ at $\mathrm{FCz}$ (left) indicates

their nonsignificant relationship $(p=.91)$. The pink shaded area represent 95\% bootstrapped confident intervals around the linear regression line. Topographical map (right) depicts the (lack of) relationship (Spearman rank coefficients) at different electrodes. Note that Spearman rank coefficients below/over $-/+.34$ are considered significant at $p=.05$ (two-tailed) (Color figure online)

slope and $\triangle \mathrm{P} 3$ as predictors and $\mathrm{AUC}$ as a criterion variable in a multiple-regression model $\left[\mathrm{AUC}=\mathrm{B}_{0}+\left(\mathrm{B}_{\mathrm{SPN} \text { slope }} \times \mathrm{SPN}\right.\right.$ slope $)+\left(\mathrm{B}_{\Delta \mathrm{P} 3} \times \Delta \mathrm{P} 3\right)+$ error]. The relationship between the rank-transformed SPN slope and $\triangle \mathrm{P} 3$ was not significant in this model, $r_{s}(32)=-.08, p=.66$. Moreover, having both the SPN slope and $\triangle \mathrm{P} 3$ in the model explained $32.8 \%$ of the variance of the AUC, $F(2,31)=7.56, p=.002$, and both the SPN slope $(\beta=-.38, p=.014)$ and the $\Delta \mathrm{P} 3(\beta=-.40, p=$ .011) uniquely predicted the AUC.

\section{Discussion}

In line with previous studies on anticipation and reward sensitivity (Benningfield et al., 2014; Weber et al., 2007), we predicted that elevated outcome-anticipation and outcome-evaluation processes should be associated with a stronger ability to delay gratification, as reflected by a stronger

preference toward larger-but-delayed rewards. Overall, our results supported this prediction at both the outcome-anticipation (SPN) and late (P3) outcome evaluation (but not at early [FRN] outcome evaluation) phases. For outcome anticipation, we demonstrated, for the first time, that individuals who had a more negative SPN slope when anticipating reward/punishment outcome (thereby having enhanced outcome-anticipation processing; Foti \& Hajcak, 2012; Fuentemilla et al., 2013) had a stronger tendency to wait for larger rewards, and forgo immediate-but-smaller rewards. This enhanced preference for larger-but-delayed rewards is consistent with previous research that manipulated anticipation-related processes (Weber et al., 2007). As discussed, after enhancing anticipation-related processes through manipulations (e.g., think about a larger-but-delayed reward before considering a smaller-but-immediate reward; Weber et al., 2007), participants in previous studies displayed an increased preference for larger-but-delayed rewards. Here, 

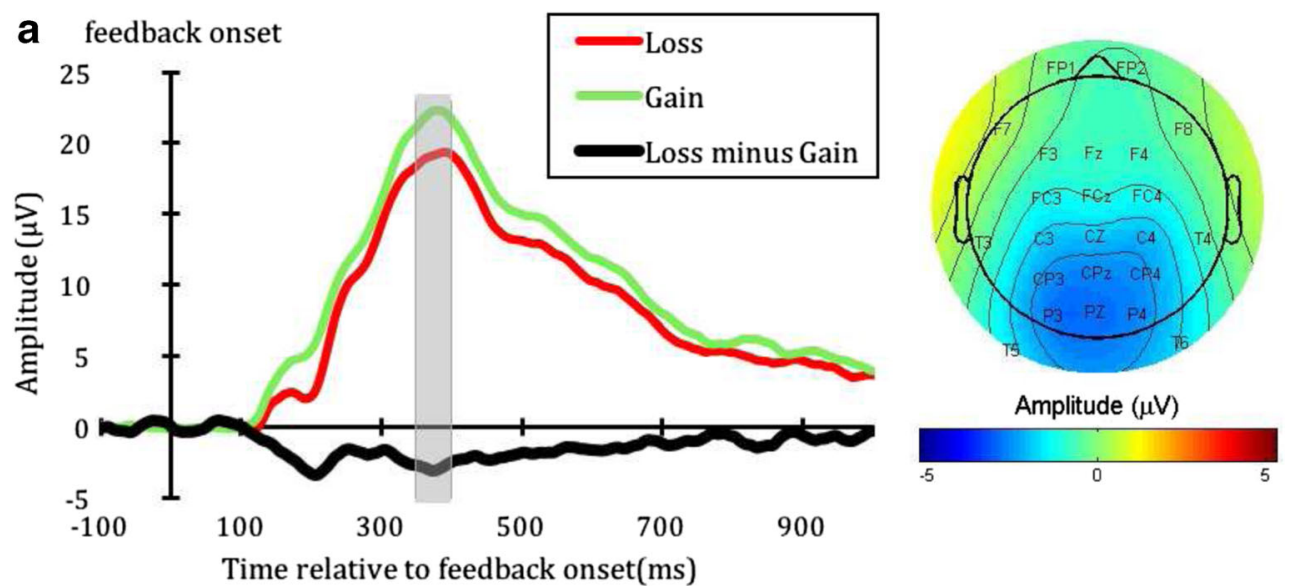

b
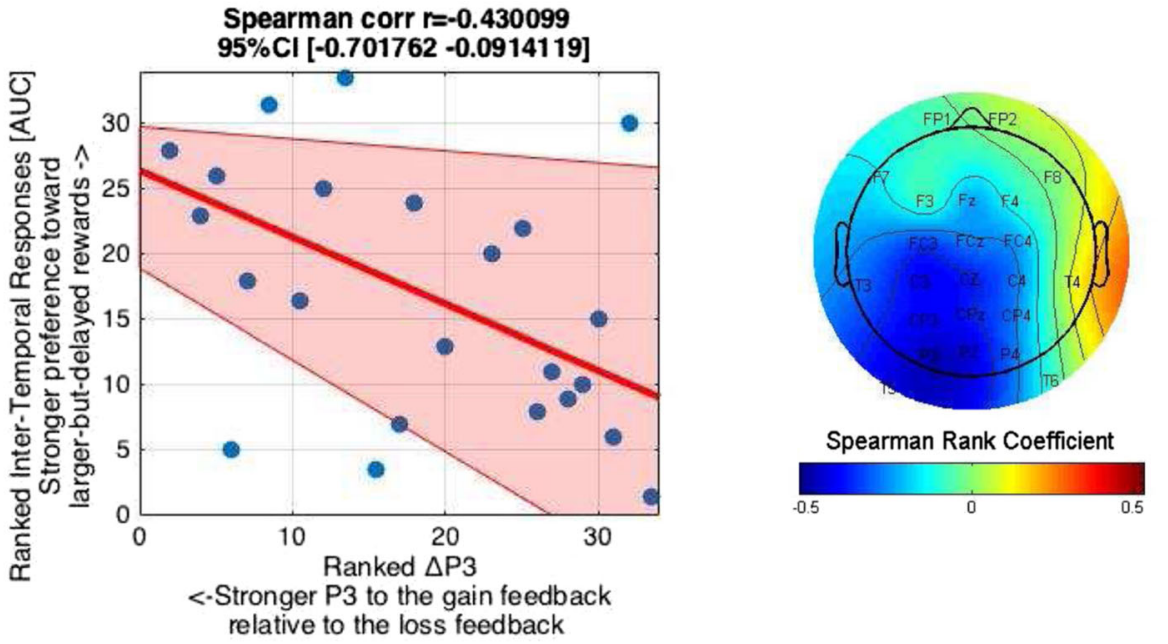

Fig. 5 The P3. a P3 waveform at Pz (left) and its topographical map (right). Topographical map shows the difference in ERP activity between the loss and gain feedback (loss minus gain) during 350 to $400 \mathrm{~ms}$ after the feedback onset (indicated by the gray area in the waveform). $\mathbf{b}$ Relationship between intertemporal responses (AUC) and $\triangle \mathrm{P} 3$ (Loss P3 minus Gain P3). A scatterplot between ranked AUC and $\Delta \mathrm{P} 3$ at $\mathrm{Pz}$ (left) indicates their significant relationship $(p=.01)$. The pink shaded

area represent $95 \%$ bootstrapped confidence intervals around the linear regression line. Topographical map (right) depicts the relationship (Spearman rank coefficients) at different electrodes, showing significant relationships $\left(r_{s}<-.34, p\right.$ 's $\left.<.05\right)$ at FC3, C3, Cz, CP3, P3, Pz, and T5. Note also that the more negative the $\Delta \mathrm{P} 3$, the greater the $\mathrm{P} 3$ amplitude in response to the gain (compared to loss) feedback (Color figure online)

instead of manipulating anticipation-related processes, we demonstrate that having a stronger outcome-anticipation (SPN) neural index during an uncertain reward/punishment situation predicts a stronger preference for larger-but-delayed

Table 1 Rank-transformed multiple regression analysis of Intertemporal responses (AUC) as predicted by outcome-anticipation (SPN slope) and outcome-evaluation $(\triangle \mathrm{P} 3)$ ERPs

\begin{tabular}{lllll}
\hline & $\mathrm{B}$ & $S E \mathrm{~B}$ & $\beta$ & $p$ \\
\hline Constant & 31.05 & 3.77 & & $<.001$ \\
SPN slope & -.36 & .14 & -.38 & .014 \\
$\Delta \mathrm{P} 3$ & -.37 & .14 & -.40 & .011 \\
\hline
\end{tabular}

Note. $R^{2}=.33(p=.002)$. Collinearity statistics (tolerance $=.995, \mathrm{VIF}=$ $1.005)$ indicated that multicollinearity between the predictors was not a concern. The $\triangle \mathrm{P} 3$ difference score is calculated by $\mathrm{P} 3$ to loss feedback minus $\mathrm{P} 3$ to gain feedback rewards on a separate intertemporal choice task. Thus, the outcome-anticipation processes, as reflected by the SPN, may modulate individual differences in the ability to delay gratification, as reflected by intertemporal responses.

With respect to outcome-evaluation ERPs, we found that enhancement in the late $(\Delta \mathrm{P} 3)$, but not the early $(\Delta \mathrm{FRN})$, outcome-evaluation time window predicted a stronger tendency to select larger-but-delayed rewards. Although both the FRN and P3 during outcome evaluation have been associated with individual differences in reward sensitivity as reflected by self-reported scales (Bress \& Hajcak, 2013; Van den Berg et al., 2011), these ERPs have been linked to different aspects of reward processing. While the FRN is typically associated with a quick, binary evaluation of bad versus good outcome (Folstein \& Van Petten, 2008; Hajcak et al., 2006; Miltner et al., 1997; Walsh \& Anderson, 2012), the P3 is more related to subsequent attentional resources captured by the motivational saliency of the 
feedback (Hu et al., 2015; Martin, 2012; Yeung \& Sanfey, 2004). According to this view, participants who preferred to wait for larger rewards in the present study (reflected by a higher AUC) allocated greater attention to gain (compared to loss) feedback, as reflected by a more negative $\Delta \mathrm{P} 3$ (which involves a loss $\mathrm{P} 3$ minus gain $\mathrm{P} 3$ difference score). From this perspective, a more negative $\triangle \mathrm{P} 3$ reflects the tendency to have a greater saliency bias toward gain (compared to loss) outcomes. This reasoning fits well with the previously asserted viewpoint that individuals who are biased toward larger-but-delayed choices are more sensitive to larger rewards and, thus, are more likely to forgo smaller-but-immediate rewards (Ballard \& Knutson, 2009; Benningfield et al., 2014; Pornpattananangkul \& Nusslock, 2016) By using ERPs, we have further demonstrated that only certain time windows of outcome evaluation drove this relationship ( $\triangle \mathrm{P} 3$, not $\triangle \mathrm{FRN})$. This timing information complements and extends previous fMRI studies showing that certain reward-related brain areas are sensitive to intertemporal responses (such as the VS and L-OFC; Benningfield et al., 2014; Boettiger et al., 2007; Samanez-Larkin et al., 2011). Our results are also in line with studies related to dopamine signaling. Specifically, Met allele carriers of the COMT gene, who typically have higher tonic dopamine levels and stronger activity in reward-related neural areas during fMRI reward tasks (Chen et al., 2004; Yacubian et al., 2007), usually express a stronger preference toward larger-but-delayed rewards (Boettiger et al., 2007; Gianotti et al., 2012; Smith \& Boettiger, 2012). Thus, a salience-bias toward gain (compared to loss) outcomes during the outcome-evaluation process, as reflected by the $\Delta \mathrm{P} 3$, may modulate individual differences in the ability to delay gratification, as reflected by intertemporal responses.

Moreover, using multiple regression we examined, for the first time, the unique versus shared contribution of outcome-anticipation (the SPN) and outcome-evaluation (the P3) processes in modulating individual differences in intertemporal responses. We found evidence for a unique, independent contribution, though in the same direction, from both the SPN and P3 in explaining intertemporal responses. This suggests that there are at least two dissociable pathways modulating individual variability in intertemporal responses, namely outcome-anticipation and outcome-evaluation processes. In combining these two processes, our model explained approximately one third of the interindividual variance in intertemporal responses. Our study, thus, highlights potentially dissociable processes (outcome anticipation vs. outcome evaluation) that may play an important role in biasing intertemporal responses. An important question for future research is whether biases in intertemporal responses among these different populations (e.g., individuals with a healthy body weight or strong financial success as compared to individuals with anorexia; Ayduk et al., 2000; Decker et al., 2014; Ersner-Hershfield, Garton, et al., 2009; Schlam et al., 2013) are driven by similar outcome-anticipation/evaluation processes.
Nonetheless, the (lack of) relationship between the $\Delta F R N$ and intertemporal responses contradicts the one previous study (Cherniawsky \& Holroyd, 2013). Participants in this study engaged in an ERP T-maze task (T. E. Baker \& Holroyd, 2009) that provided four types of feedback: (1) immediate high-gain, (2) immediate low-gain, (3) delayed (6 months later) high gain, and (4) delayed low gain. Their $\triangle$ FRNs were scored as the difference in EEG activity between feedbacks of different reward magnitude (low gain minus high gain) during the FRN window separately for immediate and delay feedback. The authors found that while individual differences in intertemporal responses did not correspond to the $\triangle F R N$ to delayed feedback, individuals who preferred smaller-but-immediate rewards had a stronger $\triangle$ FRN to immediate feedback. These findings contradict our null-significant correlation between $\triangle F R N$ to immediate feedback and intertemporal responses and the proposed link between elevated reward sensitivity and a greater preference for larger-but-delayed rewards discussed thus far.

We argue that this discrepancy may be due in part to Cherniawsky and Holroyd's use of immediate low-gain versus immediate high-gain feedback in calculating the $\triangle F R N$ (as opposed to immediate loss vs. immediate gain feedback in our study). In their study, an immediate low-gain feedback was not the worst feedback; it was still better than a delayed low-gain feedback. Given the context dependence of the FRN (Holroyd, Larsen, \& Cohen, 2004), interpretation of the immediate low-gain feedback may vary between individuals. People who are sensitive to rewards, for instance, may primarily view an immediate low-gain feedback as better than a delayed lowgain feedback. Because the two immediate feedbacks are interpreted as "wins," the difference in response to them would be diminished, leading to a smaller immediate FRN for those who are more sensitive to rewards. Thus, if people who prefer larger-but-delayed rewards are more sensitive to reward, as we have suggested, they would have a smaller immediate FRN than people who prefer smaller-but-immediate rewards. As such, the results from Cherniawsky and Holroyd may actually support our hypothesis. Given our use of immediate loss vs gain feedback in the current study (thereby avoiding the problem of interpreting the negative feedback between individuals), our nonsignificant correlation between $\triangle F R N$ and intertemporal responses supports our observation of Cherniawsky and Holroyd's study. Yet future investigation that has all loss, low-gain, and high-gain feedback in the same task is needed to fully investigate this observation. Second, as noted in their paper, "the difference [FRN] waves peaked outside of the window, during the period of the P300 ERP component" (Cherniawsky \& Holroyd, 2013, p. 40). This suggests that their ERP task could not differentiate between the FRN and P3 effects. Accordingly, their frontal FRN was confounded by the frontal P3, making it difficult to interpret whether the effect was driven by the FRN or P3. In contrast, our study showed a clear 
dissociation where the difference between the gain and loss feedback at FCz was apparent at the FRN window, but not at the $\mathrm{P} 3$ window. In fact, we found a clear relationship between the AUC and $\triangle \mathrm{P} 3$ at $\mathrm{Pz}$ that was significantly stronger than the relationship between the AUC and $\triangle \mathrm{FRN}$ at FCz. Thus, we argue that the relationship between intertemporal responses and outcome-evaluation ERPs is driven by the P3, as opposed to the FRN.

One limitation of the current experiment is that, during the outcome-anticipation period in the doors task, there was no control condition in which the feedback was not motivationally significant (e.g., waiting for feedback that was not associated with losses and gains). The SPN is typically observed when individuals are waiting to receive feedback on their performance (Brunia, van Boxtel, et al., 2011; Damen \& Brunia, 1987), regardless of the motivational significance of the feedback. However, motivationally significant feedback (e.g., loss vs. gain) enhances the SPN more than nonmotivationally significant feedback (Brunia, Hackley, et al., 2011). Thus, it is unclear whether the relationship between the SPN and intertemporal responses observed in the current study is specific to the outcome anticipation of motivationally significant feedback (loss vs. gain feedback), or the outcome anticipation of any feedback related to one's action. Having a control condition in future research may also help researchers distinguish between two types of anticipatory processes proposed to be involved in intertemporal responses. The first type relates to the experience of dreading or savoring the outcome during the anticipation period, and the physiological arousal associated with such states (Berns et al., 2007; Frederick et al., 2003; Loewenstein, 2000). This may be reflected, for instance, by the enhancement of the SPN during a motivationally significant condition relative to a control condition. The second type relates to a more general anticipatory process involving thoughts about the future (Ersner-Hershfield et al., 2011; Ersner-Hershfield, Garton, et al., 2009; Ersner-Hershfield, Wimmer, et al., 2009; Peters \& Büchel, 2011). For example, participants typically express a stronger preference toward larger-but-delayed rewards after being reminded of unrelated plans they have on the day of delayed reward delivery (e.g., vacation in Paris in 3 months; Peters \& Büchel, 2010) and after being exposed to an age-morphed version of participants' future selves via a virtual reality (Ersner-Hershfield et al., 2011). This type of anticipation of the nominal outcome is perhaps better captured by the SPN during a control condition. We decided to not include the control condition here to limit the task length, given that fatigue attenuates reward-related EEG data (Boksem \& Tops, 2008). Future research with a control condition is needed to test these possibilities. Nonetheless, even without the control condition, we should still be able to conclude from the current design that individual differences in outcome-anticipation are related to intertemporal responses.
Another limitation is related to our definition of reward sensitivity. We interpreted enhanced outcome-evaluation ERPs as reflecting enhanced reward sensitivity based on previous psychological studies of individual-differences. Specifically, previous research has linked outcome-evaluation ERPs with self-reported reward sensitivity, clinical symptoms characterized by elevated (e.g., hypomania) or diminished (e.g., depression) reward sensitivity, reward-learning performance and genetics related to reward sensitivity (e.g., Met allele of the COMT gene; e.g., Balconi \& Crivelli, 2010; Bress et al., 2013; Foti \& Hajcak, 2012; Krämer et al., 2008; Mason, O'Sullivan, Bentall, \& ElDeredy, 2012; Smillie et al., 2011; Van den Berg et al., 2011). Our definition of reward sensitivity, however, may not perfectly fit with the definition of reward sensitivity provided by economists, who initially conceptualized and proposed the link between reward sensitivity and intertemporal responses (Berns et al., 2007; Frederick et al., 2003; Loewenstein, 2000). This makes it complicated to directly map findings from the present study to theories in economics. In particular, economists typically view reward sensitivity in terms of individual differences in curvature of a utility function (Pine et al., 2009). People with high reward sensitivity are those who are less likely to diminish subjective values (utility) of objective values (reward magnitude) as the magnitude of the objective values increases, and are characterized by having less concave and more linear mapping between their subjective and objective values. Unfortunately, using one reward value in our doors task, and using adaptive measures in our intertemporal choice task, did not allow us to model individual's utility concavity as an economic index for reward sensitivity. Future ERP research is needed to confirm whether the outcome-evaluation FRN and P3 are related to utility concavity in a separate task. Additionally, future research should combine the intertemporal discounting function and utility function within the same Softmax model, and investigate their interaction on ERPs, similar to a previous fMRI study (Pine et al., 2009).

To conclude, both elevated outcome-anticipation and outcome-evaluation processes modulated individual differences in the ability to delay gratification, as reflected by preference for later-but-larger rewards over smaller-but-immediate rewards. We also found that only certain time windows (the P3 but not FRN) are sensitive to the relationship between outcome evaluation and intertemporal responses. Moreover, outcome-anticipation (the SPN) and outcome-evaluation (the P3) processes uniquely modulated individual differences in intertemporal responses. Altogether, we believe findings from the present study further our understanding of individual differences in neural-cognitive processes, including anticipation-related processing (SPN) and a saliency bias toward gain, compared to loss, outcomes (P3) that modulate people's ability to delay gratification.

Acknowledgements This work was supported by NIH Grant T32 NS047987 to NP. R.N.'s contribution to this work was supported by 
National Institute of Mental Health (NIMH) Grant R01 MH100117-01 and R01 MH077908-01A1 as well as a Young Investigator Grant from the Ryan Licht Sang Bipolar Foundation and the Chauncey and Marion D. McCormick Family Foundation. The authors thank Darren Tanner for assisting with ERP simulation (see Supplementary pp. 2-4) and Nicolas Escoffier for helpful discussion.

\section{References}

Ahn, W.-Y., Rass, O., Fridberg, D. J., Bishara, A. J., Forsyth, J. K., Breier, A., ... \& O"Donnell, B. F. (2011). Temporal discounting of rewards in patients with bipolar disorder and schizophrenia. Journal of Abnormal Psychology, 120(4), 911-921. doi:10.1037/a0023333

Ainslie, G. (1975). Specious reward: A behavioral theory of impulsiveness and impulse control. Psychological Bulletin, 82(4), 463-496. doi: $10.1037 / \mathrm{h} 0076860$

Ayduk, O., Mendoza-Denton, R., Mischel, W., Downey, G., Peake, P. K., \& Rodriguez, M. (2000). Regulating the interpersonal self: Strategic self-regulation for coping with rejection sensitivity. Journal of Personality and Social Psychology, 79(5), 776-792.

Baker, T. E., \& Holroyd, C. B. (2009). Which way do I go? Neural activation in response to feedback and spatial processing in a virtual T-maze. Cerebral Cortex, 19(8), 1708-1722. doi:10.1093/cercor/ bhn223

Baker, K. S., Piriyapunyaporn, T., \& Cunnington, R. (2012). Neural activity in readiness for incidental and explicitly timed actions. Neuropsychologia, 50(5), 715-722. doi:10.1016/j. neuropsychologia.2011.12.026

Balconi, M., \& Crivelli, D. (2010). FRN and P300 ERP effect modulation in response to feedback sensitivity: The contribution of punishmentreward system (BIS/BAS) and Behaviour Identification of action. Neuroscience Research, 66(2), 162-172. doi:10.1016/j.neures. 2009.10.011

Ballard, K., \& Knutson, B. (2009). Dissociable neural representations of future reward magnitude and delay during temporal discounting. NeuroImage, 45(1), 143-150. doi:10.1016/j.neuroimage.2008.11. 004

Bartels, D. M., \& Urminsky, O. (2011). On intertemporal selfishness: How the perceived instability of identity underlies impatient consumption. Journal of Consumer Research, 38(1), 182-198. doi:10. 1086/658339

Benningfield, M. M., Blackford, J. U., Ellsworth, M. E., Samanez-Larkin, G. R., Martin, P. R., Cowan, R. L., \& Zald, D. H. (2014). Caudate responses to reward anticipation associated with delay discounting behavior in healthy youth. Developmental Cognitive Neuroscience, 7, 43-52. doi:10.1016/j.den.2013.10.009

Berns, G. S., Laibson, D., \& Loewenstein, G. (2007). Intertemporal choice-Toward an integrative framework. Trends in Cognitive Sciences, 11(11), 482-488. doi:10.1016/j.tics.2007.08.011

Blackburn, M., Mason, L., Hoeksma, M., Zandstra, E. H., \& El-Deredy, W. (2012). Delay discounting as emotional processing: An electrophysiological study. Cognition and Emotion, 26(8), 1459-1474. doi: 10.1080/02699931.2012.673478

Böcker, K. E., Brunia, C. M., \& Berg-Lenssen, M. C. (1994). A spatiotemporal dipole model of the stimulus preceding negativity (SPN) prior to feedback stimuli. Brain Topography, 7(1), 71-88. doi:10. 1007/BF01184839

Boettiger, C. A., Mitchell, J. M., Tavares, V. C., Robertson, M., Joslyn, G., D'Esposito, M., \& Fields, H. L. (2007). Immediate reward bias in humans: Fronto-parietal networks and a role for the catechol-omethyltransferase $158 \mathrm{Val} / \mathrm{Val}$ genotype. The Journal of Neuroscience, 27(52), 14383-14391. doi:10.1523/jneurosci.255107.2007
Boksem, M. A. S., \& Tops, M. (2008). Mental fatigue: Costs and benefits. Brain Research Reviews, 59(1), 125-139. doi:10.1016/j.brainresrev. 2008.07.001

Bress, J. N., Foti, D., Kotov, R., Klein, D. N., \& Hajcak, G. (2013). Blunted neural response to rewards prospectively predicts depression in adolescent girls. Psychophysiology, 50(1), 74-81. doi:10. 1111/j.1469-8986.2012.01485.x

Bress, J. N., \& Hajcak, G. (2013). Self-report and behavioral measures of reward sensitivity predict the feedback negativity. Psychophysiology, 50(7), 610-616. doi:10.1111/psyp.12053

Bress, J. N., Smith, E., Foti, D., Klein, D. N., \& Hajcak, G. (2012). Neural response to reward and depressive symptoms in late childhood to early adolescence. Biological Psychology, 89(1), 156-162. doi:10. 1016/j.biopsycho.2011.10.004

Brunia, C. H. M., Hackley, S. A., van Boxtel, G. J., Kotani, Y., \& Ohgami, Y. (2011). Waiting to perceive: Reward or punishment? Clinical Neurophysiology, 122(5), 858-868. doi:10.1016/j.clinph. 2010.12.039

Brunia, C. H. M., van Boxtel, G. J. M., \& Böcker, K. B. E. (2011). Negative slow waves as indices of anticipation: The bereitschaftspotential, the contingent negative variation, and the stimulus preceding negativity. In E. S. Kappenman \& S. J. Luck (Eds.), The Oxford handbook of event-related potential components. doi:10.1093/oxfordhb/9780195374148.013.0108

Chapman, L. J., \& Chapman, J. P. (1987). The measurement of handedness. Brain and Cognition, 6(2), 175-183. doi:10.1016/02782626(87)90118-7

Chen, J., Lipska, B. K., Halim, N., Ma, Q. D., Matsumoto, M., Melhem, S., ... \& Weinberger, D. R. (2004). Functional analysis of genetic variation in catechol-o-methyltransferase (COMT): Effects on mRNA, protein, and enzyme activity in postmortem human brain. The American Journal of Human Genetics, 75(5), 807-821. doi:10. $1086 / 425589$

Cherniawsky, A., \& Holroyd, C. (2013). High temporal discounters overvalue immediate rewards rather than undervalue future rewards: An event-related brain potential study. Cognitive, Affective, \& Behavioral Neuroscience, 13(1), 36-45. doi:10.3758/s13415-0120122-x

Clayson, P. E., Baldwin, S. A., \& Larson, M. J. (2013). How does noise affect amplitude and latency measurement of event-related potentials (ERPs)? A methodological critique and simulation study. Psychophysiology, 50(2), 174-186. doi:10.1111/psyp.12001

Cohen, J., \& Polich, J. (1997). On the number of trials needed for P300. International Journal of Psychophysiology, 25(3), 249-255. doi:10. 1016/s0167-8760(96)00743-x

Conover, W. J., \& Iman, R. L. (1981). Rank transformations as a bridge between parametric and nonparametric statistics. The American Statistician, 35(3), 124-129. doi:10.1080/00031305.1981. 10479327

Damen, E. J. P., \& Brunia, C. H. M. (1987). Changes in heart rate and slow brain potentials related to motor preparation and stimulus anticipation in a time estimation task. Psychophysiology, 24(6), 700 713. doi:10.1111/j.1469-8986.1987.tb00353.x

Damen, E. J. P., \& Brunia, C. H. M. (1994). Is a stimulus conveying taskrelevant information a sufficient condition to elicit a stimuluspreceding negativity? Psychophysiology, 31(2), 129-139. doi:10. 1111/j.1469-8986.1994.tb01033.x

Decker, J. H., Figner, B., \& Steinglass, J. E. (2014). On weight and waiting: Delay discounting in anorexia nervosa pretreatment and sosttreatment. Biological Psychiatry. doi:10.1016/j.biopsych.2014. 12.016

Delorme, A., \& Makeig, S. (2004). EEGLAB: An open source toolbox for analysis of single-trial EEG dynamics including independent component analysis. Journal of Neuroscience Methods, 134(1), 921. doi:10.1016/j.jneumeth.2003.10.009 
Donchin, E., \& Coles, M. G. (1988). Is the P300 component a manifestation of context updating? Behavioral and Brain Sciences, 11(3), 357-427. doi:10.1017/s0140525x00058027

Donkers, F. C., Nieuwenhuis, S., \& van Boxtel, G. J. (2005). Mediofrontal negativities in the absence of responding. Brain Research: Cognitive Brain Research, 25(3), 777-787. doi:10. 1016/j.cogbrainres.2005.09.007

Du, W. J., Green, L., \& Myerson, J. (2002). Cross-cultural comparisons of discounting delayed and probabilistic rewards. Psychological Record, 52(4), 479-492.

Dunning, J. P., \& Hajcak, G. (2007). Error-related negativities elicited by monetary loss and cues that predict loss. Neuroreport, 18(17), 18751878. doi:10.1097/WNR.0b013e3282f0d50b

Ersner-Hershfield, H., Garton, M. T., Ballard, K., Samanez-Larkin, G. R., \& Knutson, B. (2009). Don't stop thinking about tomorrow: Individual differences in future self-continuity account for saving. Judgment and Decision Making, 4(4), 280-286.

Ersner-Hershfield, H., Goldstein, D. G., Sharpe, W. F., Fox, J., Leo Yeykelis, Carstensen, L. L., ... \& Bailenson, J. N. (2011). Increasing saving behavior through age-progressed renderings of the future self. Journal of Marketing Research, 48(SPL), S23-S37. doi:doi:10.1509/jmkr.48.SPL.S23

Ersner-Hershfield, H., Wimmer, G. E., \& Knutson, B. (2009). Saving for the future self: Neural measures of future self-continuity predict temporal discounting. Social Cognitive and Affective Neuroscience, 4(1), 85-92. doi:10.1093/scan/nsn042

Folstein, J. R., \& Van Petten, C. (2008). Influence of cognitive control and mismatch on the N2 component of the ERP: A review. Psychophysiology, 45(1), 152-170. doi:10.1111/j.1469-8986.2007. 00602.x

Foti, D., \& Hajcak, G. (2009). Depression and reduced sensitivity to nonrewards versus rewards: Evidence from event-related potentials. Biological Psychology, 81(1), 1-8. doi:10.1016/j.biopsycho.2008. 12.004

Foti, D., \& Hajcak, G. (2012). Genetic variation in dopamine moderates neural response during reward anticipation and delivery: Evidence from event-related potentials. Psychophysiology, 49(5), 617-626. doi:10.1111/j.1469-8986.2011.01343.x

Foti, D., Kotov, R., Klein, D., \& Hajcak, G. (2011). Abnormal neural sensitivity to monetary gains versus losses among adolescents at risk for depression. Journal of Abnormal Child Psychology, 39(7), 913924. doi:10.1007/s10802-011-9503-9

Frederick, S., Loewenstein, G., \& O'Donoghue, T. (2003). Time discounting and time preference: A critical review. In G. Loewenstein, D. Read, \& R. Baumeister (Eds.), Time and decision: Economic and psychological perspectives on intertemporal choice (pp. xiii, 13-86, 569). New York, NY: Russell Sage Foundation.

Fuentemilla, L., Cucurell, D., Marco-Pallares, J., Guitart-Masip, M., Moris, J., \& Rodriguez-Fornells, A. (2013). Electrophysiological correlates of anticipating improbable but desired events. NeuroImage, 78, 135-144. doi:10.1016/j.neuroimage.2013.03.062

Gianotti, L. R. R., Figner, B., Ebstein, R. P., \& Knoch, D. (2012). Why some people discount more than others: Baseline activation in the dorsal PFC mediates the link between COMT genotype and impatient choice. Frontiers in Neuroscience, 6. doi:10.3389/fnins.2012. 00054

Goldstein, R. Z., Cottone, L. A., Jia, Z., Maloney, T., Volkow, N. D., \& Squires, N. K. (2006). The effect of graded monetary reward on cognitive event-related potentials and behavior in young healthy adults. International Journal of Psychophysiology, 62(2), 272279. doi:10.1016/j.ijpsycho.2006.05.006

Green, L., \& Myerson, J. (2004). A discounting framework for choice with delayed and probabilistic rewards. Psychological Bulletin, 130(5), 769-792. doi:10.1037/0033-2909.130.5.769

Hajcak, G., Moser, J. S., Holroyd, C. B., \& Simons, R. F. (2006). The feedback-related negativity reflects the binary evaluation of good versus bad outcomes. Biological Psychology, 71(2), 148-154. doi: 10.1016/j.biopsycho.2005.04.001

Hajcak, G., Moser, J. S., Holroyd, C. B., \& Simons, R. F. (2007). It's worse than you thought: The feedback negativity and violations of reward prediction in gambling tasks. Psychophysiology, 44(6), 905912. doi:10.1111/j.1469-8986.2007.00567.x

Ho, M. Y., Mobini, S., Chiang, T. J., \& Bradshaw, C. M. (1999). Theory and method in the quantitative analysis of 'impulsive choice' behaviour: Implications for psychopharmacology. Psychopharmacology, 146(4), 362-372. doi:10.1007/p100005482

Holroyd, C. B., Larsen, J. T., \& Cohen, J. D. (2004). Context dependence of the event-related brain potential associated with reward and punishment. Psychophysiology, 41(2), 245-253. doi:10.1111/j.14698986.2004.00152.x

Holroyd, C. B., Nieuwenhuis, S., Yeung, N., Nystrom, L., Mars, R. B., Coles, M. G. H., \& Cohen, J. D. (2004). Dorsal anterior cingulate cortex shows $\mathrm{fMRI}$ response to internal and external error signals. Nature Neuroscience, 7(5), 497-498. Retrieved from http://www. nature.com/neuro/journal/v7/n5/suppinfo/nn1238_S1.html

Holroyd, C. B., Nieuwenhuis, S., Yeung, N., \& Cohen, J. D. (2003). Errors in reward prediction are reflected in the event-related brain potential. NeuroReport: For rapid communication of neuroscience research, 14(18), 2481-2484. doi:10.1097/00001756-20031219000037

Hu, X., Pornpattananangkul, N., \& Nusslock, R. (2015). Executive control- and reward-related neural processes associated with the opportunity to engage in voluntary dishonest moral decision making. Cognitive, Affective, \& Behavioral Neuroscience, 15(2), 475 491. doi:10.3758/s13415-015-0336-9

Johnson, M. W., \& Bickel, W. K. (2002). Within-subject comparison of real and hypothetical money rewards in delay discounting. Journal of the Experimental Analysis of Behavior, 77(2), 129-146. doi:10. 1901/jeab.2002.77-129

Kalenscher, T., \& Pennartz, C. M. A. (2008). Is a bird in the hand worth two in the future? The neuroeconomics of intertemporal decisionmaking. Progress in Neurobiology, 84(3), 284-315. doi:10.1016/j. pneurobio.2007.11.004

Kirby, K. (2009). One-year temporal stability of delay-discount rates. Psychonomic bulletin \& review, 16(3), 457-462. doi:10.3758/ PBR.16.3.457

Kotani, Y., \& Aihara, Y. (1999). The effect of stimulus discriminability on stimulus-preceding negativities prior to instructive and feedback stimuli. Biological Psychology, 50(1), 1-18. doi:10.1016/S03010511(98)00047-7

Kotani, Y., Kishida, S., Hiraku, S., Suda, K., Ishii, M., \& Aihara, Y. (2003). Effects of information and reward on stimulus-preceding negativity prior to feedback stimuli. Psychophysiology, 40(5), 818-826. doi:10.1111/1469-8986.00082

Kotani, Y., Ohgami, Y., Ishiwata, T., Arai, J.-I., Kiryu, S., \& Inoue, Y. (2015). Source analysis of stimulus-preceding negativity constrained by functional magnetic resonance imaging. Biological Psychology, 111, 53-64. doi:10.1016/j.biopsycho.2015.08.005

Kotani, Y., Ohgami, Y., Kuramoto, Y., Tsukamoto, T., Inoue, Y., \& Aihara, Y. (2009). The role of the right anterior insular cortex in the right hemisphere preponderance of stimulus-preceding negativity (SPN): An fMRI study. Neuroscience Letters, 450(2), 75-79. doi:10.1016/j.neulet.2008.11.032

Krämer, U. M., Büttner, S., Roth, G., \& Münte, T. F. (2008). Trait aggressiveness modulates neurophysiological correlates of laboratoryinduced reactive aggression in humans. Journal of Cognitive Neuroscience, 20(8), 1464-1477. doi:10.1162/jocn.2008.20103

Lagorio, C. H., \& Madden, G. J. (2005). Delay discounting of real and hypothetical rewards III: Steady-state assessments, forced-choice trials, and all real rewards. Behavioural Processes, 69(2), 173187. doi:10.1016/j.beproc.2005.02.003 
Loewenstein, G. (1988). Frames of mind in intertemporal vhoice. Management Science, 34(2), 200-214. doi:10.1287/mnsc.34.2.200

Loewenstein, G. (1996). Out of control: Visceral influences on behavior. Organizational Behavior and Human Decision Processes, 65(3), 272-292. doi:10.1006/obhd.1996.0028

Loewenstein, G. (2000). Emotions in economic theory and economic behavior. The American Economic Review, 90(2), 426-432.

Luck, S. J. (2014). An introduction to the event-related potential technique. Cambridge: MIT Press.

Luft, C. D. B. (2014). Learning from feedback: The neural mechanisms of feedback processing facilitating better performance. Behavioural Brain Research, 261, 356-368. doi:10.1016/j.bbr.2013.12.043

Marco-Pallares, J., Cucurell, D., Münte, T. F., Strien, N., \& RodriguezFornells, A. (2011). On the number of trials needed for a stable feedback-related negativity. Psychophysiology, 48(6), 852-860. doi:10.1111/j.1469-8986.2010.01152.x

Martin, R. S. (2012). Event-related potential studies of outcome processing and feedback-guided learning. Frontiers in Human Neuroscience, 6. doi:10.3389/fnhum.2012.00304

Masaki, H., Takeuchi, S., Gehring, W. J., Takasawa, N., \& Yamazaki, K. (2006). Affective-motivational influences on feedback-related ERPs in a gambling task. Brain Research, 1105(1), 110-121. doi:10.1016/ j.brainres.2006.01.022

Mason, L., O’Sullivan, N., Bentall, R. P., \& El-Deredy, W. (2012). Better than I thought: Positive evaluation bias in hypomania. PLOS ONE, 7(10), e47754. doi:10.1371/journal.pone.0047754

Mason, L., O’Sullivan, N., Blackburn, M., Bentall, R., \& El-Deredy, W. (2012). I want it now! Neural correlates of hypersensitivity to immediate reward in hypomania. Biological Psychiatry, 71(6), 530537. doi:10.1016/j.biopsych.2011.10.008

Miltner, W. H. R., Braun, C. H., \& Coles, M. G. H. (1997). Event-related brain potentials following incorrect feedback in a time-estimation task: Evidence for a 'generic' neural system for error detection. Journal of Cognitive Neuroscience, 9(6), 788-798. doi:10.1162/ jocn.1997.9.6.788

Morís, J., Luque, D., \& Rodríguez-Fornells, A. (2013). Learning-induced modulations of the stimulus-preceding negativity. Psychophysiology, 50(9), 931-939. doi:10.1111/psyp.12073

Myerson, J., Green, L., \& Warusawitharana, M. (2001). Area under the curve as a measure of discounting. Journal of the Experimental Analysis of Behavior, 76(2), 235-243. doi:10.1901/jeab.2001.76235

Nieuwenhuis, S., Aston-Jones, G., \& Cohen, J. D. (2005). Decision making, the P3, and the locus coeruleus-norepinephrine system. Psychological Bulletin, 131(4), 510-532. doi:10.1037/0033-2909. 131.4.510

Odum, A. L. (2011). Delay discounting: Trait variable? Behavioural Processes, 87(1), 1-9. doi:10.1016/j.beproc.2011.02.007

Ohgami, Y., Kotani, Y., Hiraku, S., Aihara, Y., \& Ishii, M. (2004). Effects of reward and stimulus modality on stimulus-preceding negativity. Psychophysiology, 41(5), 729-738. doi:10.1111/j.1469-8986.2004. 00203.x

Ohmura, Y., Takahashi, T., Kitamura, N., \& Wehr, P. (2006). Threemonth stability of delay and probability discounting measures. Experimental and Clinical Psychopharmacology, 14(3), 318-328. doi:10.1037/1064-1297.14.3.318

Pernet, C. R., Wilcox, R., \& Rousselet, G. A. (2012). Robust correlation analyses: False positive and power validation using a new open source matlab toolbox. Frontiers in Psychology, 3, 606. doi:10. 3389/fpsyg.2012.00606

Peters, J., \& Büchel, C. (2010). Episodic future thinking reduces reward delay discounting through an enhancement of prefrontalmediotemporal interactions. Neuron, 66(1), 138-148. doi:10.1016/ j.neuron.2010.03.026
Peters, J., \& Büchel, C. (2011). The neural mechanisms of inter-temporal decision-making: Understanding variability. Trends in Cognitive Sciences, 15(5), 227-239. doi:10.1016/j.tics.2011.03.002

Pine, A., Seymour, B., Roiser, J. P., Bossaerts, P., Friston, K. J., Curran, H. V., \& Dolan, R. J. (2009). Encoding of marginal utility across time in the human brain. The Journal of Neuroscience, 29(30), 9575-9581. doi:10.1523/jneurosci.1126-09.2009

Plichta, M. M., \& Scheres, A. (2014). Ventral-striatal responsiveness during reward anticipation in ADHD and its relation to trait impulsivity in the healthy population: A meta-analytic review of the fMRI literature. Neuroscience \& Biobehavioral Reviews, 38, 125-134. doi:10.1016/j.neubiorev.2013.07.012

Pornpattananangkul, N., \& Nusslock, R. (2015). Motivated to win: Relationship between anticipatory and outcome reward-related neural activity. Brain and Cognition, 100, 21-40. doi:10.1016/j.bandc. 2015.09.002

Pornpattananangkul, N., \& Nusslock, R. (2016). Willing to wait: Elevated reward-processing EEG activity associated with a greater preference for larger-but-delayed rewards. Neuropsychologia, 91, 141-162. doi:10.1016/j.neuropsychologia.2016.07.037

Pronin, E., Olivola, C. Y., \& Kennedy, K. A. (2008). Doing unto future selves as you would do unto others: Psychological distance and decision making. Personality and Social Psychology Bulletin, 34(2), 224-236. doi:10.1177/0146167207310023

Proudfit, G. H. (2015). The reward positivity: From basic research on reward to a biomarker for depression. Psychophysiology, 52(4), 449-459. doi:10.1111/psyp.12370

Qu, C., Huang, Y., Wang, Y., \& Huang, Y. (2013). The delay effect on outcome evaluation: Results from an event-related potential study. Frontiers in Human Neuroscience, 7. doi:10.3389/fnhum.2013. 00748

Rachlin, H., Raineri, A., \& Cross, D. (1991). Subjective probability and delay. Journal of the Experimental Analysis of Behavior, 55(2), 233 244. doi:10.1901/jeab.1991.55-233

Rass, O., Ahn, W. Y., \& O’Donnell, B. F. (2015). Resting-state EEG, impulsiveness, and personality in daily and nondaily smokers. Clinical Neurophysiology. doi:10.1016/j.clinph.2015.05.007

Reynolds, B. (2006). A review of delay-discounting research with humans: Relations to drug use and gambling. Behavioural Pharmacology, 17(8), 651-667. doi:10.1097/FBP. 0b013e3280115f99

Samanez-Larkin, G. R., Mata, R., Radu, P. T., Ballard, I. C., Carstensen, L. L., \& McClure, S. M. (2011). Age differences in striatal delay sensitivity during intertemporal choice in healthy adults. Frontiers in Neuroscience, 5. doi:10.3389/fnins.2011.00126

Samuelson, P. A. (1937). Some aspects of the pure theory of capital. The Quarterly Journal of Economics, 51(3), 469-496. doi:10.2307/ 1884837

Schlam, T. R., Wilson, N. L., Shoda, Y., Mischel, W., \& Ayduk, O. (2013). Preschoolers' delay of gratification predicts their body mass 30 years later. The Journal of Pediatrics, 162(1), 90-93. doi:10. 1016/j.jpeds.2012.06.049

Schultz, W. (2010). Subjective neuronal coding of reward: Temporal value discounting and risk. European Journal of Neuroscience, 31(12), 2124-2135. doi:10.1111/j.1460-9568.2010.07282.x

Sellitto, M., Ciaramelli, E., \& di Pellegrino, G. (2010). Myopic discounting of future rewards after medial orbitofrontal damage in humans. The Journal of Neuroscience, 30(49), 16429-16436. doi: 10.1523/jneurosci.2516-10.2010

Shamosh, N. A., DeYoung, C. G., Green, A. E., Reis, D. L., Johnson, M. R., Conway, A. R. A., ... \& Gray, J. R. (2008). Individual differences in delay discounting: Relation to intelligence, working memory, and anterior prefrontal cortex. Psychological Science, 19(9), 904-911. doi:10.1111/j.1467-9280.2008.02175.x

Smillie, L. D., Cooper, A. J., \& Pickering, A. D. (2011). Individual differences in reward-prediction-error: Extraversion and feedback- 
related negativity. Social Cognitive and Affective Neuroscience, 6(5), 646-652. doi:10.1093/scan/nsq078

Smith, C., \& Boettiger, C. (2012). Age modulates the effect of COMT genotype on delay discounting behavior. Psychopharmacology, 222(4), 609-617. doi:10.1007/s00213-012-2653-9

Tanner, D., Morgan-Short, K., \& Luck, S. J. (2015). How inappropriate high-pass filters can produce artifactual effects and incorrect conclusions in ERP studies of language and cognition. Psychophysiology, 52(8), 997-1009. doi:10.1111/psyp. 12437

Tanner, D., Norton, J. J. S., Morgan-Short, K., \& Luck, S. J. (2016). On high-pass filter artifacts (they're real) and baseline correction (it's a good idea) in ERP/ERMF analysis. Journal of Neuroscience Methods, 266, 166-170. doi:10.1016/j.jneumeth.2016.01.002

Tom, S. M., Fox, C. R., Trepel, C., \& Poldrack, R. A. (2007). The neural basis of loss aversion in decision-making under risk. Science, 315(5811), 515-518. doi:10.1126/science.1134239

Tversky, A., \& Kahneman, D. (1992). Advances in prospect theory: Cumulative representation of uncertainty. Journal of Risk and Uncertainty, 5(4), 297-323. doi:10.1007/BF00122574

Van Boxtel, G., Ullsperger, M., \& Falkenstein, M. (2004). The use of the subtraction technique in the psychophysiology of response inhibition and conflict. In M. Ullsperger \& M. Falkenstein (Eds.), Errors, conflicts, and the brain: Current opinions on performance monitoring (pp. 219-225). Leipzig: Max Planck Institute.

Van den Berg, I., Franken, I. H. A., \& Muris, P. (2011). Individual differences in sensitivity to reward: Association with electrophysiological responses to monetary gains and losses. Journal of Psychophysiology, 25(2), 81-86. doi:10.1027/0269-8803/a000032
Verboven, S., \& Hubert, M. (2005). LIBRA: A MATLAB library for robust analysis. Chemometrics and Intelligent Laboratory Systems, 75(2), 127-136. doi:10.1016/j.chemolab.2004.06.003

Walsh, M. M., \& Anderson, J. R. (2012). Learning from experience: Event-related potential correlates of reward processing, neural adaptation, and behavioral choice. Neuroscience and Biobehavioral Reviews, 36(8), 1870-1884. doi:10.1016/j.neubiorev.2012.05.008

Weaver, B., \& Wuensch, K. L. (2013). SPSS and SAS programs for comparing Pearson correlations and OLS regression coefficients. Behavior Research Methods, 45(3), 880-895.

Weber, E. U., Johnson, E. J., Milch, K. F., Chang, H., Brodscholl, J. C., \& Goldstein, D. G. (2007). Asymmetric discounting in intertemporal choice: A query-theory account. Psychological Science, 18(6), 516523. doi:10.1111/j.1467-9280.2007.01932.x

Weller, R. E., Cook, E. W., Avsar, K. B., \& Cox, J. E. (2008). Obese women show greater delay discounting than healthy-weight women. Appetite, 51(3), 563-569. doi:10.1016/j.appet.2008.04.010

Williams, E. J. (1959). The comparison of regression variables. Journal of the Royal Statistical Society Series B:Statistical Methodology, 21(2), 396-399.

Yacubian, J., Sommer, T., Schroeder, K., Glascher, J., Kalisch, R., Leuenberger, B., ... \& Buchel, C. (2007). Gene-gene interaction associated with neural reward sensitivity. Proceedings of the National Academy of Sciences, 104(19), 8125-8130. doi:10.1073/ pnas.0702029104

Yeung, N., \& Sanfey, A. G. (2004). Independent coding of reward magnitude and valence in the human brain. The Journal of Neuroscience, 24(28), 6258-6264. doi:10.1523/jneurosci.4537-03. 2004 